\title{
Regular and chaotic dynamics of non-spherical bodies. Zeldovich's pancakes and emission of very long gravitational waves
}

\author{
G. S. BIS NOVATYI - KOGAN ${ }^{1,2} \dagger$ AND O. YU. TSUPKO $\mathbf{O}^{1,2}$ \\ ${ }^{1}$ Space Research Institute of Russian Academy of Sciences, Profsoyuznaya 84/32, Moscow \\ 117997, Russia \\ ${ }^{2}$ National Research Nuclear University MEPhI (Moscow Engineering Physics Institute), \\ Kashirskoe Shosse 31, Moscow 115409, Russia
}

(Received 22 July 2018)

In this paper we review a recently developed approximate method for investigation of dynamics of compressible ellipsoidal figures. Collapse and subsequent behaviour are described by a system of ordinary differential equations for time evolution of semi-axes of a uniformly rotating, three-axis, uniform-density ellipsoid. First, we apply this approach to investigate dynamic stability of non-spherical bodies. We solve the equations that describe, in a simplified way, the Newtonian dynamics of a self-gravitating non-rotating spheroidal body. We find that, after loss of stability, a contraction to a singularity occurs only in a pure spherical collapse, and deviations from spherical symmetry prevent the contraction to the singularity through a stabilizing action of nonlinear non-spherical oscillations. The development of instability leads to the formation of a regularly or chaotically oscillating body, in which dynamical motion prevents the formation of the singularity. We find regions of chaotic and regular pulsations by constructing a Poincar diagram. A real collapse occurs after damping of the oscillations because of energy losses, shock wave formation or viscosity. We use our approach to investigate approximately the first stages of collapse during the large scale structure formation. The theory of this process started from ideas of Ya. B. Zeldovich, concerning the formation of strongly non-spherical structures during nonlinear stages of the development of gravitational instability, known as 'Zeldovich's pancakes'. In this paper the collapse of non-collisional dark matter and the formation of pancake structures are investigated approximately. Violent relaxation, mass and angular momentum losses are taken into account phenomenologically. We estimate an emission of very long gravitational waves during the collapse, and discuss the possibility of gravitational lensing and polarization of the cosmic microwave background by these waves.

\section{Introduction}

Observed Universe is non-homogeneous at different scales. Perturbations of density lead to gravitational collapse of matter and formation of structures of different sizes: stars, galaxies, large scale structure. Many of astrophysical objects are essentially non-spherical, and their formation and evolution can be approximately described as a behaviour of selfgravitating ellipsoidal bodies in the frame of Newtonian gravity.

Theory of ellipsoidal figures was developed in classical works of Newton, Maclaurin, Jacobi, Poincare, Chandrasechar. The systematic analysis of ellipsoidal figures of equilibrium is presented in the book of Chandrasekhar (1969). Different types of incompressible

† Email address for correspondence: gkogan@iki.rssi.ru, tsupko@iki.rssi.ru 
ellipsoids are investigated there on a basis of virial equations, as well as their dynamical and secular stability.

The shape of ellipsoid is determined by sizes of its three semi-axes. Non-rotating figures always take the spherical form, all semi-axes are equal. In the presence of rotation the spheroid (axially-symmetrical ellipsoid with two equal semi-axes) is formed (Maclaurin spheroid). If the angular momentum of rotation is large enough, development of different kinds of instabilities lead to formation of a three-axis ellipsoid, with rotation around the small axis (Jacobi ellipsoid). Incompressible spheroid can be secularly or dynamically unstable for transition to three-axis ellipsoid. The points of the onset of the bar-mode dynamical, and secular instabilities of the Maclaurin spheroid, for its transition into the Jacobi ellipsoid, are found in the book Chandrasekhar (1969).

Approach based on ellipsoidal description allows to investigate the time evolution of a body considering behaviour of its semi-axes. First of all, its initial collapse can be described, which is a central problem in formation of astrophysical objects. In the case of the collapse compressible ellipsoidal objects are considered. Lynden-Bell $(1964,1965)$ applied the Chandrasechar's virial tensor method for the rotating, self-gravitating spheroid of pressure-free gas, and had shown the growth of non-axisymmetric perturbations during the collapse. It was also discussed that the slowly shrinking Maclaurin spheroid will enter the Jacobi series if it shrinks slowly enough for the dissipative mechanisms to be operative. Zeldovich (1964) considered the dynamics of rotating dust ellipsoids with linear dependence of velocities on coordinates, when the problem is solved exactly. There are numerical investigations of collapsing pressureless spheroids in papers of Lvnden-Bell (1964) and Lin, Mestel \& Shu (1965). In the paper of Rosensteel \& Tran (1991) the virial equations for rotating Riemann ellipsoids of incompressible fluid are demonstrated to form a Hamiltonian dynamical system. There is a detailed description of ellipsoid model of rotating stars in papers of Lai. Rasio \& Shapiro (1993, 1994a, b). Using a variational principle, they had derived and investigated equations of the evolution of a compressible Riemann-S ellipsoid, incorporating viscous dissipation and gravitational radiation. Solutions of these approximate equations permitted to obtain equilibrium models, and to investigate their dynamical and secular stability. The point of instability of compressible Maclaurin spheroids is found in Lai, Rasio \& Shapiro (1993) and Shapiro (2004). There is a wide ranging review of this topic in the paper of Lynden-Bell (1996) in memory of Chandrasekhar.

In the present paper we review the developed approximate model for consideration of dynamics of collapsing ellipsoidal figures. Present review is based on the papers of Bisnovatyi-Kogan (2004), Bisnovatyi-Kogan \& Tsupko (2005), Bisnovatyi-Kogan \& Tsupko (2008a). We derive and solve equations of the dynamical behavior in a simple model of a compressible uniformly rotating three-axis ellipsoid. The ordinary differential equations for ellipsoid semi-axes evolution in time are obtained with help of variation of the Lagrange function of ellipsoid. In our model a motion along three axes takes place in the gravitational field of a uniform density ellipsoid, with account of rotation, represented by centrifugial forces, and the isotropic pressure, represented in an approximate non-differential way.

Our model can be used for investigation of star dynamics, and also for the first stages of formation of large scale structure consisted from dark matter, with appropriate approximations. Starting from section 2, we apply our model for investigation of dynamic stability of non-spherical stars. We show, that deviation from the spherical symmetry in a non-rotating star with zero angular momentum leads to formation of a regularly or chaotically oscillating body, in which dynamical motion prevents the formation of the singularity. The non-spherical star without dissipative processes never will reach a singu- 
larity in the Newtonian dynamics. Therefore collapse to a singularity is connected with a secular type of instability, even without rotation. Starting from section 7, we consider approximate model for large scale structure formation. We estimate emission of a very long gravitational waves during a collapse of the dark matter, and discuss a possibility of gravitational lensing, and polarisation of CMB by these waves.

Recently Vandervoort (2011) models the galaxy as a heterogeneous ellipsoid with an arbitrary stratification of the density distribution, and represent the mean motions of the stars in terms of a velocity field that sustains that density distribution consistently with the equation of continuity. The virial equations are reduced to a closed system of equations of motion that govern the semi-axes of the characteristic ellipsoid. The semiaxes of an ellipsoid characterizing the size and shape of the galaxy are functions of the time, whereas the stratification of the density does not change. In the second paper (Vandervoort 2014) author describes an application of the model to an investigation of chaotic behaviour in axisymmetric pulsations of non-rotating, spherically symmetric configurations and of rotating, axisymmetric configurations. See also papers of Rodrigues (2014), Sharif \& Zaeem Ul Haq Bhatti (2013), Sharif \& Zaeem Ul Haq Bhatti (2014).

\section{Dynamic stability of non-spherical stars}

Dynamic stability of spherical stars is determined by an average adiabatic power

$$
\gamma=\left.\frac{\partial \log P}{\partial \log \rho}\right|_{S} .
$$

For a density distribution

$$
\rho=\rho_{0} \varphi\left(\frac{m}{M}\right),
$$

the star in the newtonian gravity is stable against dynamical collapse when (Zeldovich \& Novikov 1967; Bisnovatyi-Kogan 1989)

$$
\int_{0}^{R}\left(\gamma-\frac{4}{3}\right) P \frac{d m}{\varphi(m / M)}>0 .
$$

Here $\rho_{0}$ is a central density, $M$ is a stellar mass, $m$ is the mass inside a Lagrangian radius $r$, so that $m=4 \pi \int_{0}^{r} \rho r^{2} d r, M=m(R), R$ is a stellar radius, $\varphi\left(\frac{m}{M}\right)$ is the Emden function corresponding to $\gamma=4 / 3$.

Let us consider equation of state $P=K \rho^{\gamma}$. Spherical star with such equation of state is stable against collapse if $\gamma>4 / 3$ and unstable at $\gamma<4 / 3$, when the instability leads to a collapse to singularity. At $\gamma=4 / 3$ there is only one equilibrium state for a given mass, at a unique value of the constant $K_{0}$. This state is neutral to the stability, and may suffer contraction to singularity at $K<K_{0}$, or expansion to infinity at $K>K_{0}$.

Collapse of a spherical, initially unstable star may be stopped only by a stiffening of the equation of state, like neutron star formation at late stages of evolution, or formation of fully ionized stellar core with $\gamma=\frac{5}{3}$ at the collapse of clouds during star formation. Without such stiffening a spherical star in the newtonian theory would collapse into a point with an infinite density (singularity).

In the presence of a rotation a star is becoming more dynamically stable against collapse. Due to the more rapid increase of a centrifugal force during contraction, in comparison with the newtonian gravitational force, collapse of a rotating star will be always stopped at finite density by centrifugal forces.

Here we show, that deviation from the spherical symmetry in a non-rotating star with zero angular momentum leads to a similar stabilization, and non-spherical star without 
dissipative processes never will reach a singularity. Therefore collapse to a singularity is connected with a secular type of instability, even without rotation.

We calculate a dynamical behavior of a non-spherical, non-rotating star after its loss of a linear stability, and investigate nonlinear stages of contraction. We use approximate system of dynamic equations, describing 3 degrees of freedom of a uniform self-gravitating compressible ellipsoidal body (Bisnovatyi-Kogan \& Tsupko 2008a). We obtain that the development of instability leads to the formation of a regularly or chaotically oscillating body, in which dynamical motion prevents the formation of the singularity. We find regions of chaotic and regular pulsations by constructing a Poincaré diagram for different values of the initial eccentricity and initial entropy. For simplicity we restrict ourself by calculating only spheroidal figures with $\gamma=\frac{4}{3}$, see also results for $\gamma=\frac{6}{5}$ in Bisnovatyi-Kogan \& Tsupko (2008a).

\section{Equations of motion for semi-axes of rotating 3-axis ellipsoid}

Here we derive equations of motion for general case of rotating 3 -axis ellipsoid and consider cases $\gamma=5 / 3$ and $\gamma=4 / 3$.

Let us consider a compressible 3 -axis ellipsoid with semi-axes $a \neq b \neq c$

$$
\frac{x^{2}}{a^{2}}+\frac{y^{2}}{b^{2}}+\frac{z^{2}}{c^{2}}=1
$$

We assume that the ellipsoid rotates uniformly with an angular velocity $\Omega$ around the axis $z$. Let us approximate the density of the matter $\rho$ in the ellipsoid as a uniform. During collapse and posterior time evolution the sizes of semi-axes $a, b, c$, angular velocity $\Omega$ and density $\rho$ are changed with time, but ellipsoid keeps ellipsoidal shape with the homogeneous distribution of density and uniform rotation.

The constant mass $m$ and total angular momentum $M$ of the uniform ellipsoid are connected with uniform density, angular velocity and semi-axes as $(V$ is the volume of the ellipsoid)

$$
m=\rho V=\frac{4 \pi}{3} \rho a b c, \quad M=\rho \Omega \int_{V}\left(x^{2}+y^{2}\right) d V=\frac{m}{5} \Omega\left(a^{2}+b^{2}\right) .
$$

We assume a linear dependence of the velocities on the coordinates in the rotating frame

$$
v_{x}=\frac{\dot{a} x}{a}, \quad v_{y}=\frac{\dot{b} y}{b}, \quad v_{z}=\frac{\dot{c} z}{c} .
$$

If an homogeneous ellipsoidal body of inviscid fluid initially has velocities that are linear functions of position, then it will remain ellipsoidal and the internal velocities will remain linear functions of position for all time provided that the density remains spatially uniform (see, for example, Lynden-Bell (1996)).

In absence of any dissipation this ellipsoid is a conservative system. To derive the equations of motion (equations describing time evolutions of semi-axes) let write a Lagrange function of the ellipsoid which consists of kinetic energy $U_{k i n}$ and potential energy $U_{p o t}$ of ellipsoid:

$$
L=U_{k i n}-U_{p o t}, \quad U_{p o t}=U_{g}+E_{t h}+U_{r o t} .
$$

The kinetic energy is written as (with using (3.3))

$$
U_{k i n}=\frac{1}{2} \rho \int_{V}\left(v_{x}^{2}+v_{y}^{2}+v_{z}^{2}\right) d V=\frac{m}{10}\left(\dot{a}^{2}+\dot{b}^{2}+\dot{c}^{2}\right) .
$$


The gravitational energy $U_{g}$ of the uniform ellipsoid is defined as (Landau \& Lifshitz 1993):

$$
U_{g}=-\frac{3 G m^{2}}{10} \int_{0}^{\infty} \frac{d u}{\sqrt{\left(a^{2}+u\right)\left(b^{2}+u\right)\left(c^{2}+u\right)}} .
$$

In case of spheroids $(a=b \neq c)$ this integral can be taken analytically, in case of ellipsoid it is expressed in elliptical integrals (see Bisnovatyi-Kogan (2004), Bisnovatyi-Kogan \& Tsupko (2005)).

The rotational energy is

$$
U_{\text {rot }}=\frac{1}{2} \rho \int_{V} v_{\text {rot }}^{2} d V=\frac{1}{2} \rho \Omega^{2} \int_{V}\left(x^{2}+y^{2}\right) d x d y d z=\frac{m}{10} \Omega^{2}\left(a^{2}+b^{2}\right) .
$$

The angular velocity $\Omega$ is changed with time, and it is more convenient to express (3.7) via $M$ which is constant in absence of dissipation. Taking into account the second expression in (3.2), we obtain the relation

$$
U_{\text {rot }}=\frac{5}{2} \frac{M^{2}}{m\left(a^{2}+b^{2}\right)} .
$$

Different form of thermal energy $E_{t h}$ are used depending on problem under consideration.

\subsection{Rotating ellipsoid, case $\gamma=5 / 3$}

First, we use the non-relativistic equation of state $P=K \rho^{\gamma}$ with polytropic index $\gamma=$ $5 / 3$. It corresponds to the case of a matter consisted of non-collisional non-relativistic dark matter particles.

The total thermal energy, with adiabatic index $n=1.5(\gamma=5 / 3)$, of non-relativistic dark matter particles in the ellipsoid is $E_{t h} \sim V^{-2 / 3} \sim(a b c)^{-2 / 3}$, and the relation between pressure $P$ and thermal energy $E_{t h}$ is $E_{t h}=\frac{3}{2} P V$. Let us write $E_{t h}$ using initial values $E_{t h, i n}, a_{i n}, b_{i n}, c_{i n}$ as

$$
E_{t h}=\frac{E_{t h, i n}\left(a_{i n} b_{i n} c_{i n}\right)^{2 / 3}}{(a b c)^{2 / 3}}=\frac{\varepsilon}{(a b c)^{2 / 3}} .
$$

We introduce here $\varepsilon$, which we will call as the entropy function. The entropy function $\varepsilon$ is constant in the conservative case, but it is variable in presence of a dissipation.

By variation of the Lagrange function we obtain the Lagrange equations of motion:

$$
\begin{gathered}
\ddot{a}=-\frac{3 G m}{2} a \int_{0}^{\infty} \frac{d u}{\left(a^{2}+u\right) \Delta}+\frac{10}{3 m} \frac{1}{a} \frac{\varepsilon}{(a b c)^{2 / 3}}+\frac{25 M^{2}}{m^{2}} \frac{a}{\left(a^{2}+b^{2}\right)^{2}}, \\
\ddot{b}=-\frac{3 G m}{2} b \int_{0}^{\infty} \frac{d u}{\left(b^{2}+u\right) \Delta}+\frac{10}{3 m} \frac{1}{b} \frac{\varepsilon}{(a b c)^{2 / 3}}+\frac{25 M^{2}}{m^{2}} \frac{b}{\left(a^{2}+b^{2}\right)^{2}}, \\
\ddot{c}=-\frac{3 G m}{2} c \int_{0}^{\infty} \frac{d u}{\left(c^{2}+u\right) \Delta}+\frac{10}{3 m} \frac{1}{c} \frac{\varepsilon}{(a b c)^{2 / 3}}, \Delta^{2}=\left(a^{2}+u\right)\left(b^{2}+u\right)\left(c^{2}+u\right) .
\end{gathered}
$$

At the right-hand side of these equations there are forces per unit ellipsoid mass. The first term is the gravitational force directed to the center of ellipsoid, the second term 
is the force of thermal pressure directed outward, and the third term is the centrifugal force directed outward.

It is easy to check that equilibrium solution of these equations are the Maclaurin spheroid and the Jacobi ellipsoid (Chandrasekhar 1969).

\subsection{Non-rotating ellipsoid, $\gamma=4 / 3$}

For investigation of dynamic stability of non-spherical bodies we consider the nonrotating compressible homogeneous ellipsoid with the equation of state $P=K \rho^{\gamma}, \gamma=$ $4 / 3$. The case $\gamma=5 / 3$ considered above is not interesting for investigations of dynamic stability, because isentropic spherical star with $\gamma=5 / 3$ always stops contraction, and never suffers collapse to singularity.

A spherical star with $\gamma=4 / 3$ collapses to singularity at small enough $K$, and we show here, how deviations from a spherical form prevent formation of any singularity. For $\gamma=4 / 3$, the thermal energy of the ellipsoid is $E_{t h} \sim V^{-1 / 3} \sim(a b c)^{-1 / 3}$, and the value

$$
\varepsilon=E_{t h}(a b c)^{1 / 3}=3\left(\frac{3 m}{4 \pi}\right)^{1 / 3} K
$$

remains constant in time. A Lagrange function of the ellipsoid is written as

$$
L=U_{k i n}-U_{p o t}, \quad U_{p o t}=U_{g}+E_{t h}, \quad E_{t h}=\frac{\varepsilon}{(a b c)^{1 / 3}} .
$$

Equations of motion describing behavior of 3 semiaxes $(a, b, c)$ is obtained from the Lagrange function (3.13) in the form

$$
\begin{gathered}
\ddot{a}=-\frac{3 G m}{2} a \int_{0}^{\infty} \frac{d u}{\left(a^{2}+u\right) \Delta}+\frac{5}{3 m} \frac{1}{a} \frac{\varepsilon}{(a b c)^{1 / 3}}, \\
\ddot{b}=-\frac{3 G m}{2} b \int_{0}^{\infty} \frac{d u}{\left(b^{2}+u\right) \Delta}+\frac{5}{3 m} \frac{1}{b} \frac{\varepsilon}{(a b c)^{1 / 3}}, \\
\ddot{c}=-\frac{3 G m}{2} c \int_{0}^{\infty} \frac{d u}{\left(c^{2}+u\right) \Delta}+\frac{5}{3 m} \frac{1}{c} \frac{\varepsilon}{(a b c)^{1 / 3}}, \\
\Delta^{2}=\left(a^{2}+u\right)\left(b^{2}+u\right)\left(c^{2}+u\right) .
\end{gathered}
$$

\section{Dynamic stabilization of non-spherical bodies against unlimited collapse, numerical results}

To obtain a numerical solution of equations (3.14), (3.15), (3.16) we write them in non-dimensional variables. Let us introduce the variables

$$
\begin{gathered}
\tilde{t}=\frac{t}{t_{0}}, \tilde{a}=\frac{a}{a_{0}}, \tilde{b}=\frac{b}{a_{0}}, \tilde{c}=\frac{c}{a_{0}}, \\
\tilde{m}=\frac{m}{m_{0}}, \tilde{\rho}=\frac{\rho}{\rho_{0}}, \tilde{U}=\frac{U}{U_{0}}, \quad \tilde{E}_{t h}=\frac{E_{t h}}{U_{0}}, \tilde{\varepsilon}=\frac{\varepsilon}{\varepsilon_{0}} .
\end{gathered}
$$


The scaling parameters $t_{0}, a_{0}, m_{0}, \rho_{0}, U_{0}, \varepsilon_{0}$ are connected by the following relations

$$
t_{0}^{2}=\frac{a_{0}^{3}}{G m_{0}}, U_{0}=\frac{G m_{0}^{2}}{a_{0}}, \rho_{0}=\frac{m_{0}}{a_{0}^{3}}, \varepsilon_{0}=U_{0} a_{0} .
$$

System of non-dimensional equations:

$$
\begin{gathered}
\ddot{a}=-\frac{3 m}{2} a \int_{0}^{\infty} \frac{d u}{\left(a^{2}+u\right) \Delta}+\frac{5}{3 m} \frac{1}{a} \frac{\varepsilon}{(a b c)^{1 / 3}}, \\
\ddot{b}=-\frac{3 m}{2} b \int_{0}^{\infty} \frac{d u}{\left(b^{2}+u\right) \Delta}+\frac{5}{3 m} \frac{1}{b} \frac{\varepsilon}{(a b c)^{1 / 3}}, \\
\ddot{c}=-\frac{3 m}{2} c \int_{0}^{\infty} \frac{d u}{\left(c^{2}+u\right) \Delta}+\frac{5}{3 m} \frac{1}{c} \frac{\varepsilon}{(a b c)^{1 / 3}}, \\
\Delta^{2}=\left(a^{2}+u\right)\left(b^{2}+u\right)\left(c^{2}+u\right) .
\end{gathered}
$$

In equations (4.2)-(4.4) only non-dimensional variables are used, and "tilde" sign is omitted for simplicity in this section. The non-dimensional Hamiltonian (or nondimensional total energy) is:

$$
\begin{gathered}
H=U_{k i n}+U_{g}+E_{t h}=\frac{m}{10}\left(\dot{a}^{2}+\dot{b}^{2}+\dot{c}^{2}\right)- \\
-\frac{3 m^{2}}{10} \int_{0}^{\infty} \frac{d u}{\sqrt{\left(a^{2}+u\right)\left(b^{2}+u\right)\left(c^{2}+u\right)}}+\frac{\varepsilon}{(a b c)^{1 / 3}} .
\end{gathered}
$$

In case of the sphere $(a=b=c, \dot{a}=\dot{b}=\dot{c})$ the non-dimensional Hamiltonian and non-dimensional equations of motion reduce to:

$$
\begin{gathered}
H=\frac{3}{10} m \dot{a}^{2}-\frac{3}{5 a}\left(m^{2}-\frac{5}{3} \varepsilon\right), \\
\ddot{a}=-\frac{1}{m a^{2}}\left(m^{2}-\frac{5}{3} \varepsilon\right) .
\end{gathered}
$$

As follows from (4.6), (4.7) for the given mass there is only one equilibrium value of $\varepsilon$

$$
\varepsilon_{e q}=\frac{3 m^{2}}{5}
$$

at which the spherical star has zero total energy, and it may have an arbitrary radius. For smaller $\varepsilon<\varepsilon_{e q}$ the sphere should contract to singularity, and for $\varepsilon>\varepsilon_{e q}$ there will be a total disruption of the star with an expansion to infinity. We solve here numerically the equations of motion for a spheroid with $a=b \neq c$, which, using (4.2)-(4.4), are written for the oblate spheroid with $k=c / a<1$ as

$$
\begin{gathered}
\ddot{a}=\frac{3}{2} \frac{m}{a^{2}\left(1-k^{2}\right)}\left[k-\frac{\arccos k}{\sqrt{1-k^{2}}}\right]+\frac{5}{3 m} \frac{1}{a} \frac{\varepsilon}{\left(a^{2} c\right)^{1 / 3}}, \\
\ddot{c}=-3 \frac{m}{a^{2}\left(1-k^{2}\right)}\left[1-\frac{k \arccos k}{\sqrt{1-k^{2}}}\right]+\frac{5}{3 m} \frac{1}{c} \frac{\varepsilon}{\left(a^{2} c\right)^{1 / 3}} ;
\end{gathered}
$$


and for the prolate spheroid $k=c / a>1$ as

$$
\begin{gathered}
\ddot{a}=-\frac{3}{2} \frac{m}{a^{2}\left(k^{2}-1\right)}\left[k-\frac{\cosh ^{-1} k}{\sqrt{k^{2}-1}}\right]+\frac{5}{3 m} \frac{1}{a} \frac{\varepsilon}{\left(a^{2} c\right)^{1 / 3}}, \\
\ddot{c}=3 \frac{m}{a^{2}\left(k^{2}-1\right)}\left[1-\frac{k \cosh ^{-1} k}{\sqrt{k^{2}-1}}\right]+\frac{5}{3 m} \frac{1}{c} \frac{\varepsilon}{\left(a^{2} c\right)^{1 / 3}} .
\end{gathered}
$$

It is convenient to introduce variables

$$
\varepsilon_{*}=\frac{5}{3} \frac{\varepsilon}{m^{2}}, \quad t_{*}=t \sqrt{m}
$$

In these variables the equations (4.9)-(4.12), (4.7) are written as (omitting subscript "*")

$$
\begin{aligned}
& \ddot{a}=\frac{3}{2 a^{2}\left(1-k^{2}\right)}\left[k-\frac{\arccos k}{\sqrt{1-k^{2}}}\right]+\frac{1}{a} \frac{\varepsilon}{\left(a^{2} c\right)^{1 / 3}}, \\
& \ddot{c}=-\frac{3}{a^{2}\left(1-k^{2}\right)}\left[1-\frac{k \arccos k}{\sqrt{1-k^{2}}}\right]+\frac{1}{c} \frac{\varepsilon}{\left(a^{2} c\right)^{1 / 3}}
\end{aligned}
$$

for the oblate spheroid $k=c / a<1$,

$$
\begin{gathered}
\ddot{a}=-\frac{3}{2 a^{2}\left(k^{2}-1\right)}\left[k-\frac{\cosh ^{-1} k}{\sqrt{k^{2}-1}}\right]+\frac{1}{a} \frac{\varepsilon}{\left(a^{2} c\right)^{1 / 3}}, \\
\ddot{c}=\frac{3}{a^{2}\left(k^{2}-1\right)}\left[1-\frac{k \cosh ^{-1} k}{\sqrt{k^{2}-1}}\right]+\frac{1}{c} \frac{\varepsilon}{\left(a^{2} c\right)^{1 / 3}}
\end{gathered}
$$

for the prolate spheroid $k=c / a>1$, and

$$
\ddot{a}=-\frac{1-\varepsilon}{a^{2}}
$$

for the sphere, where the equilibrium corresponds to $\varepsilon_{e q}=1$. Near the spherical shape we should use expansions around $k=1$, what leads to equations of motion valid for both oblate and prolate cases

$$
\begin{aligned}
& \ddot{a}=-\frac{1-\varepsilon}{a^{2}}+\left(\frac{\varepsilon}{3}+\frac{3}{5}\right) \frac{1-k}{a^{2}}, \\
& \ddot{c}=-\frac{1-\varepsilon}{a^{2}}+\left(\frac{4 \varepsilon}{3}-\frac{4}{5}\right) \frac{1-k}{a^{2}} .
\end{aligned}
$$

In these variables the total energy is written as

and omitting "*" we have

$$
H_{*}=\frac{H}{m^{2}}
$$

$$
\begin{aligned}
& H=\frac{\dot{a}^{2}}{5}+\frac{\dot{c}^{2}}{10}-\frac{3}{5 a} \frac{\arccos k}{\sqrt{1-k^{2}}}+\frac{3}{5} \frac{\varepsilon}{\left(a^{2} c\right)^{1 / 3}}, \quad \text { (oblate) } \\
& H=\frac{\dot{a}^{2}}{5}+\frac{\dot{c}^{2}}{10}-\frac{3}{5 a} \frac{\cosh ^{-1} k}{\sqrt{k^{2}-1}}+\frac{3}{5} \frac{\varepsilon}{\left(a^{2} c\right)^{1 / 3}}, \quad \text { (prolate) }
\end{aligned}
$$




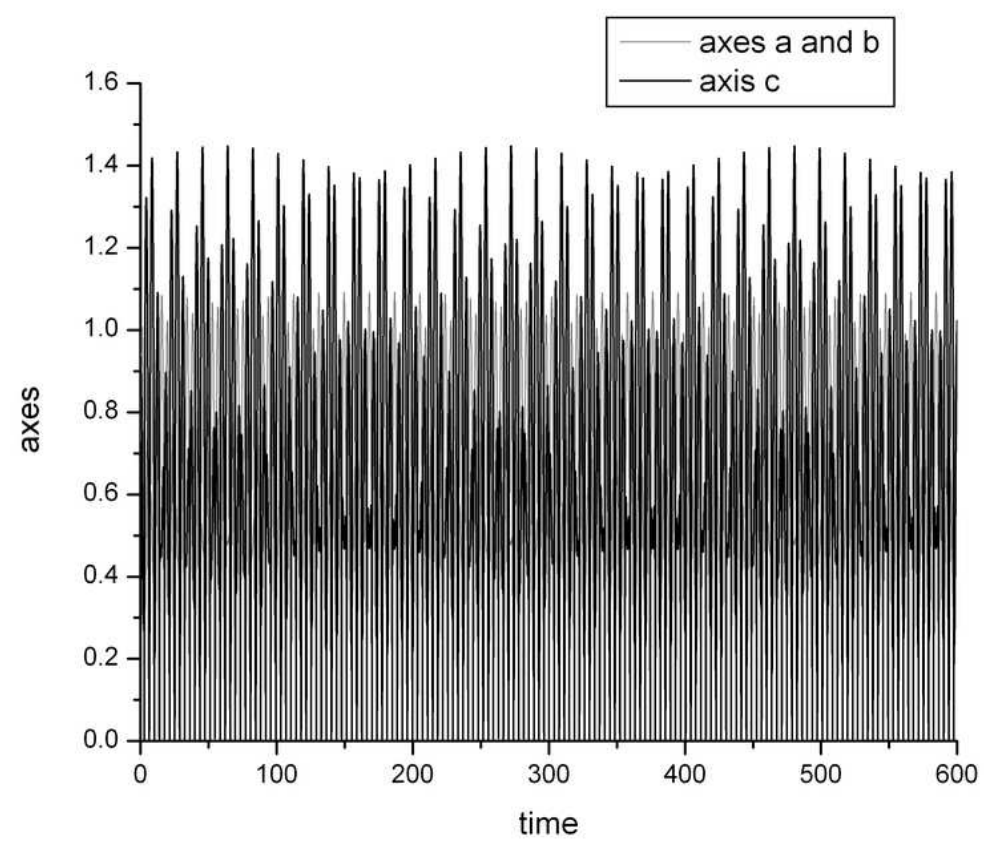

Figure 1. Example of regular motion of spheroid with $\gamma=4 / 3, H=-1 / 5, \varepsilon=2 / 3$. This motion corresponds to full line on the Poincaré map in Fig 4

$$
\begin{gathered}
H=\frac{3 \dot{a}^{2}}{10}-\frac{3}{5 a}(1-\varepsilon), \quad \text { (sphere) } \\
H=\frac{\dot{a}^{2}}{5}+\frac{\dot{c}^{2}}{10}-\frac{3}{5 a}\left(1+\frac{\delta}{3}+\frac{2 \delta^{2}}{15}\right)+\frac{3 \varepsilon}{5 a}\left(1+\frac{\delta}{3}+\frac{2 \delta^{2}}{9}\right), \\
\delta=1-k, \quad \text { (around the sphere), }|\delta| \ll 1 .
\end{gathered}
$$

Solution of the system of equations (4.9)-(4.12) was performed for initial conditions at $t=0: \dot{c}_{0}=0$, different values of initial $a_{0}, \dot{a}_{0}, k_{0}$, and different values of the constant parameter $\varepsilon$. The results of our numerical calculations are

(i) sphere $(a=b=c)$ :

$\varepsilon=1$ - total energy equals to zero $(H=0)$, radius is arbitrary,

$\varepsilon<1$ - spherical star collapses to a singularity,

$\varepsilon>1$ - disruption of star with expansion to infinity;

(ii) spheroid $(a=b \neq c)$ :

at $\varepsilon \geqslant 1$ the total energy is $H \geqslant 0$ - disruption of star with expansion to infinity, at $\varepsilon<1$ the total energy is $H<0$ - the oscillatory regime is established. In the oscillatory regime the dynamical motion prevents the formation of the singularity. The type of oscillatory regime depends on initial conditions, and may be represented either by regular periodic oscillations, or by chaotic behavior. Examples of two types of such oscillations are represented in Figs 12 (regular, periodic) and in Fig 3 (chaotic). For rigorous separation between these kinds of oscillations we use a method developed by Poincaré (Lichtenberg \& Lieberman 1983). The same approach is used in recent papers of Vandervoort (2011, 2014). 


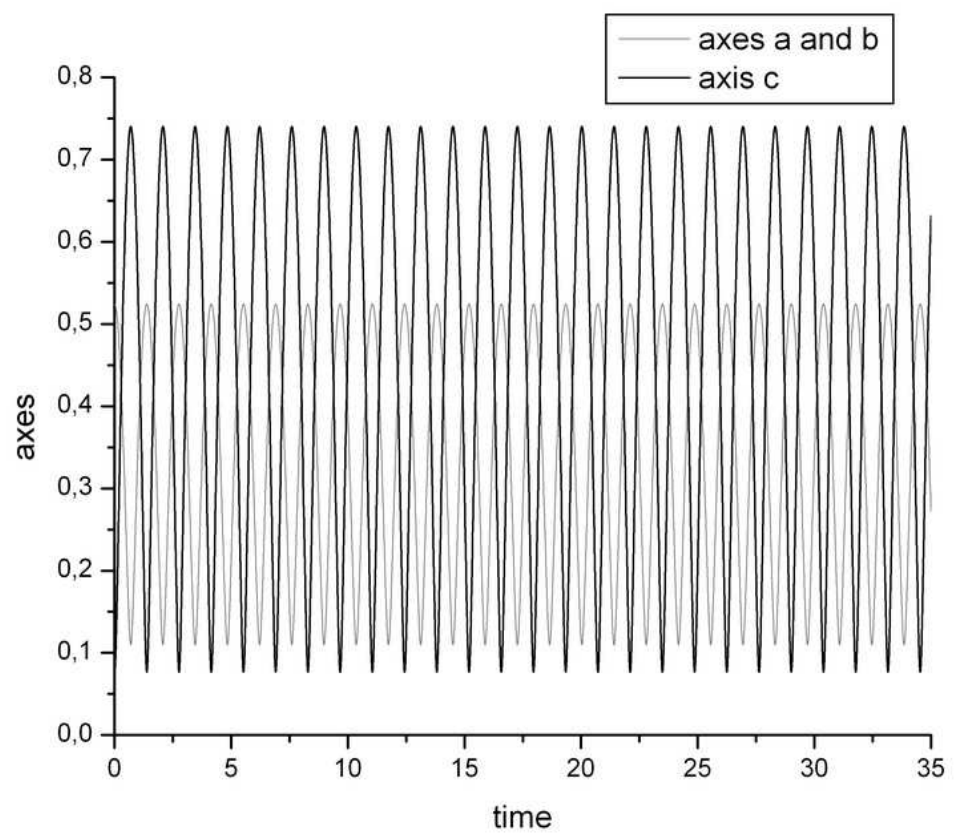

FiguRE 2. Example of regular motion of spheroid with $\gamma=4 / 3, H=-1 / 5, \varepsilon=2 / 3$. This motion corresponds to the point inside the regular region on the Poincaré map in Fig 4.

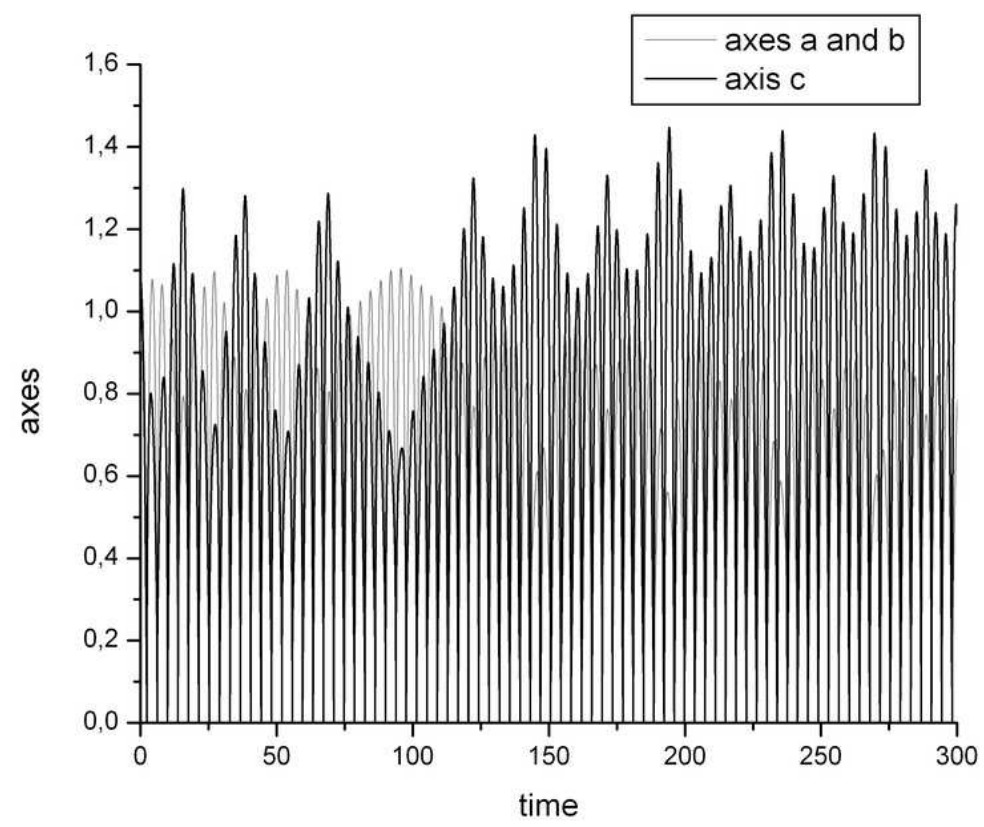

FiguRE 3. Example of chaotic motion of spheroid with $\gamma=4 / 3, H=-1 / 5, \varepsilon=2 / 3$. This motion corresponds to gray points on the Poincaré map in Fig 5 . 


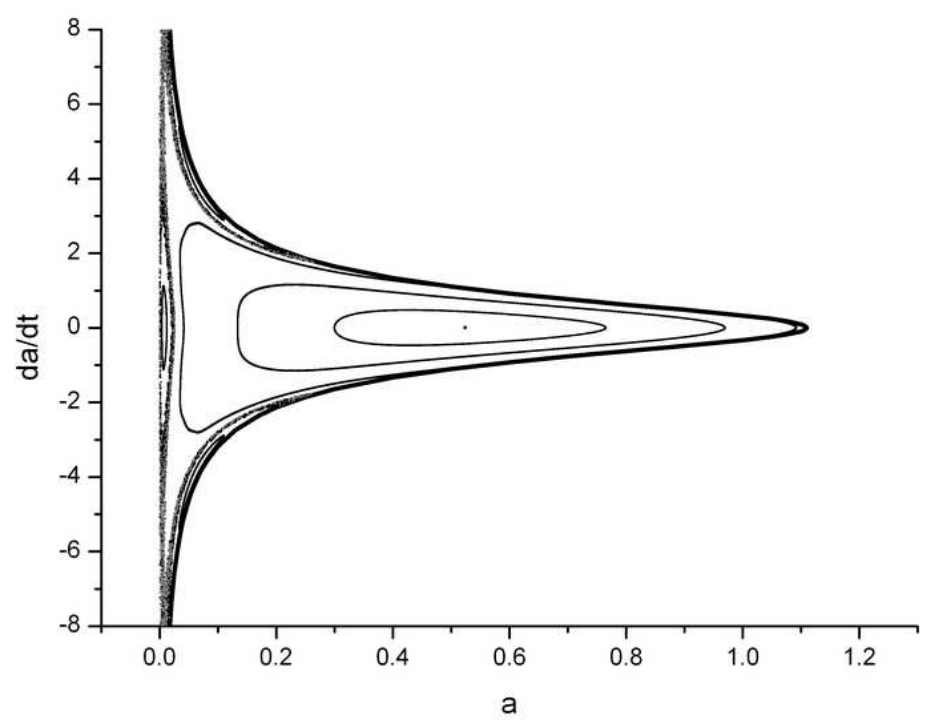

Figure 4. The Poincaré map for five regular and two chaotic trajectories in case of $\gamma=4 / 3$, $H=-1 / 5, \varepsilon=2 / 3$. The $(a, \dot{a})$ values are taken in the minimum of c. Full black line is the bounding curve. The point inside the regular region corresponds to coherent oscillations with the same period for $a$ and $c$ values, represented in Fig.2.

\section{Investigation of regular and chaotic oscillations via the Poincaré sections}

To investigate regular and chaotic dynamics we use the method of Poincaré section (Lichtenberg \& Lieberman 1983), and obtain the Poincaré map for different values of the total energy $H$. Let us consider a spheroid with semi-axes $a=b \neq c$. This system has two degrees of freedom. Therefore in this case the phase space is four-dimensional: $a, \dot{a}$, $c, \dot{c}$. If we choose, at given $\varepsilon$, a value of the Hamiltonian $H_{0}$, we fix a three-dimensional energy surface $H(a, \dot{a}, c, \dot{c})=H_{0}$. During the integration of the equations (4.9)-(4.12) which preserve the constant $H$, we fix moments $t_{i}$, when $\dot{c}=0$. At these moments there are only two independent values (i.g. $a$ and $\dot{a}$ ), because the value of $c$ is determined uniquely from the relation for the hamiltonian at constant $H$.

For the same values of $H$ and $\varepsilon$ we solve equations of motion (4.9)-(4.12) at initial $\dot{c}=0$, and different $a, \dot{a}$. For each integration we put the points on the plane $(a, \dot{a})$ at the moments $t_{i}$. These points are the intersection points of the trajectories on the three-dimensional energy surface with a two-dimensional plane $\dot{c}=0$, called a Poincaré section.

For each fixed combination of $\varepsilon, H$ we get the Poincaré map, represented in Figs 4 , 5 , 6. 7. Condition $\dot{c}=0$ splits in two cases, of a minimum and of a maximum of $c$. The Poincaré maps are drawing separately, either for the minimum, or for the maximum of $c$, and both maps lead to identical results. The regular oscillations are represented by closed lines on the Poincaré map, and chaotic behavior fills regions of finite square with dots. These regions are separated from the regions of the regular oscillations by separatrix line. 


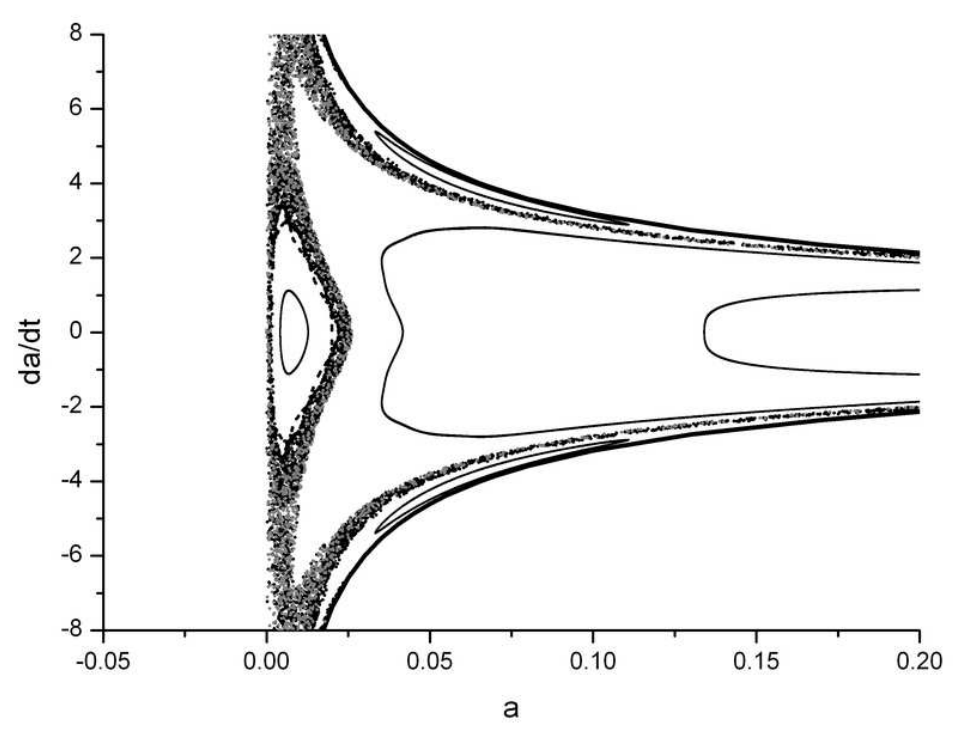

FiguRE 5. Zoom of previous figure.

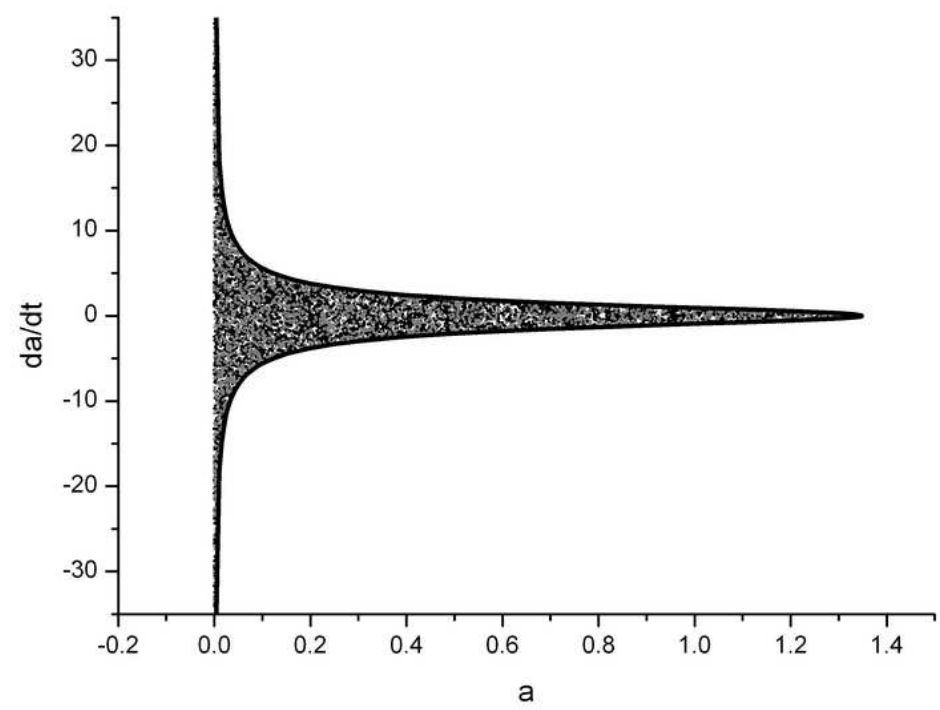

Figure 6. The Poincaré map for two chaotic trajectories in case of $\gamma=4 / 3, H=-1 / 2$, $\varepsilon=1 / 6$. The $(a, \dot{a})$ values are taken in the minimum of c. Full black line is the bounding curve.

\section{Dynamic stabilization of non-spherical bodies against unlimited collapse, discussion}

The main result following from our calculations is the indication to a degenerate nature of formation of a singularity in unstable newtonian self-gravitating gaseous bodies. Only 


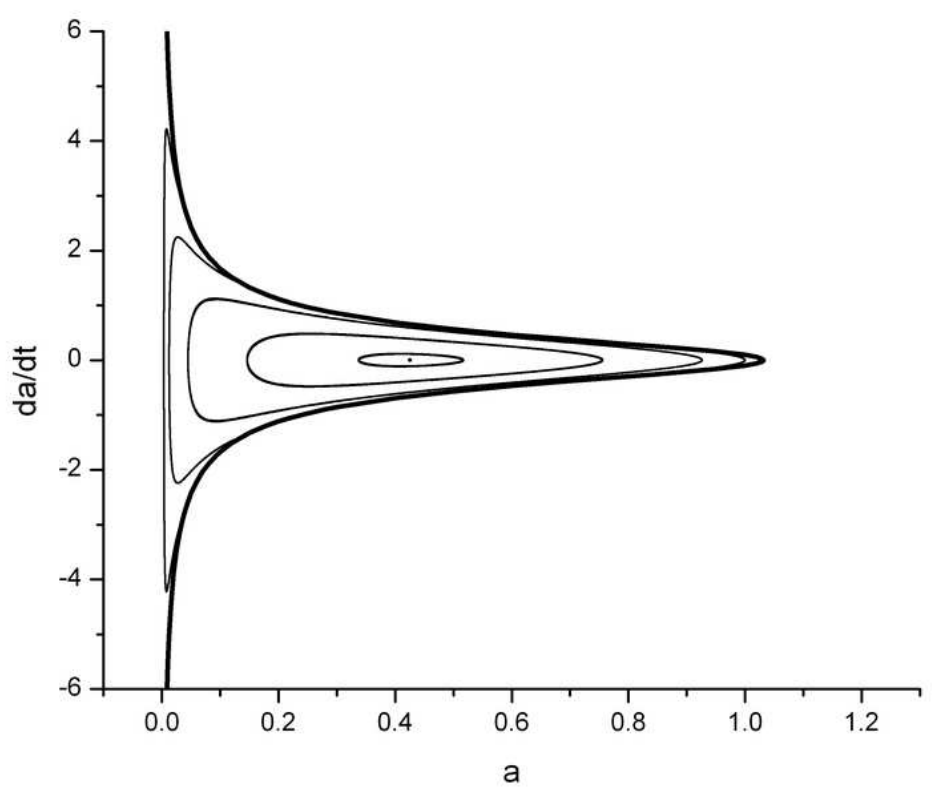

Figure 7. The Poincaré map for six regular trajectories in the case of $\gamma=4 / 3, H=-3 / 50$, $\varepsilon=9 / 10$. The $(a, \dot{a})$ values are taken in the minimum of $\mathrm{c}$. Full black line is the bounding curve. The point inside the regular region corresponds to coherent oscillations with the same period for $a$ and $c$ values, similar to those represented in Fig 2

pure spherical models can collapse to singularity, but any kind of nonsphericity leads to nonlinear stabilization of the collapse by a dynamic motion, and formation of regularly or chaotically oscillating body. This conclusion is valid for all unstable equations of state, namely, for adiabatic with $\gamma<4 / 3$. In addition to the case with $\gamma=4 / 3$, we have calculated the dynamics of the model with $\gamma=6 / 5$, and have obtained similar results.

Note, that region of chaotic behavior on the Poincaré map is gradually increasing for $\gamma=4 / 3$ with decreasing of the entropy $\varepsilon$ and the total energy $H$. At $\varepsilon=9 / 10$ and $H=-3 / 50$ we have found only regular oscillations (Fig [7), at $\varepsilon=2 / 3$ and $H=-1 / 5$ both kind of oscillations are present (Figs, 45), and only chaotic behavior is found at $\varepsilon=1 / 6$ and $H=-1 / 2$ (Fig 6). We connect (Bisnovatyi-Kogan \& Tsupko 2005) this chaotic behavior with development of anisotropic instability, when radial velocities strongly exceed the transversal ones (Antonov 1973; Fridman \& Polyachenko 1985).

In reality a presence of dissipation leads to damping of these oscillations, and to final collapse of nonrotating model, when total energy of the body is negative. In the case of core-collapse supernova the main dissipation is due to emission of neutrino. The time of the neutrino losses is much larger than the characteristic time of the collapse, so we may expect that the collapse leads to formation of a neutron star where nonspherical modes are excited and exist during several seconds after the collapse. In addition to the damping due to neutrino emission the shock waves will be generated, determining highly variable energy losses during the oscillations. Besides viscosity and radiation which can damp the ellipsoid-like motions and allow collapse, there is the possibility that inertial interactions with higher order modes that must be present in the real stratified bodies may cause an inertial cascade and drain the energy from the sec- 
ond order modes an a non-secular way that does not depend on dissipative coefficients. It is very seductive to connect chaotic oscillations with highly variable emission observed in the prompt gamma ray emission of cosmic gamma ray bursts Mazets et al. 1981). A presence of rotation and magnetic field strongly complicate the picture of the core-collapse supernova explosion (Ardelian. Bisnovatyi-Kogan \& Moiseenko 2005; Moiseenko, Bisnovatyi-Kogan \& Ardeljan 2005).

In this paper we consider in details only spheroidal bodies. In reality the spheroid will become a triaxial ellipsoid during the motion. In addition to spheroids we calculate many variants with triaxial figures (see Bisnovatyi-Kogan \& Tsupko (2005)). Qualitatively we obtain the same results for ellipsoids: no singularity was reached for any $\varepsilon>0$, and establishing oscillatory (regular or chaotic) regime under negative total energy prevents the collapse. However, in the case of the ellipsoid with semi-axes $a \neq b \neq c$ we have a system with three degrees of freedom and six-dimensional phase space. Therefore we could not carry out rigorous investigation of regular and chaotic types of motion by the constructing Poincaré map as it was done for spheroid with two degrees of freedom, and restricted ourselfs by a description of the spheroidal case.

In the frame of a general relativity dynamic stabilization against collapse by nonlinear nonspherical oscillations cannot be universal. When the size of the body approaches gravitational radius no stabilization is possible at any $\gamma$. Nevertheless, the nonlinear stabilization may happen at larger radii, so after damping of the oscillations the star would collapse to the black hole. Due to development of nonspherical oscillations there is a possibility for emission of gravitational waves during the collapse of nonrotating bodies with the intensity similar to rotating bodies, or even larger.

\section{Zeldovich's pancakes and large scale structure of Universe}

One of the most important problem in modern cosmology is a formation of large scale structure of the Universe. Observations of large scale structure of Universe demonstrate strong inhomogeneity in density distribution. Zeldovich (1970a, b has investigated the fragmentation of a homogeneous medium under the action of gravitation. It has been shown that an arbitrary perturbation in medium with zero pressure leads to formation of strongly non-spherical structures (discs), and only pure spherical perturbations lead to collapse into the point. These discs are known now as 'Zeldovich's pancakes'.

Depending on the medium properties, two types of pancakes can be formed:

(i) During contraction of the barionic gas the collapse is stopped by the pressure, the shock wave is formed. Behind the shock front the cooling, fragmentation and formation of vortexes occur, what is necessary for formation of spiral galaxies with large angular momentum.

(ii) During collapse of a non-collisional dark matter the pancake is also formed, but no shocks are forming at the stage of maximal contraction. Medium is non-collisional, so after initial the collapse, subsequent oscillations occur. The particles fly through each other many times, and mixing in the phase space occurs - so called violent relaxation. The system reaches quasi-equilibrium state via violent relaxation (Lynden-Bell 1967) and phase mixing during the most rapid dynamic stages. Established equilibrium state has a form, depending on the angular momentum of rotation, and sizes depending on the final values of energy and entropy.

Subsequent nonlinear evolution and interaction between pancakes lead to formation of a very complicated large scale structure of matter in the Universe observed in the sky. It was shown by numerical simulations in two-dimensional space by Doroshkecich et al (1980). The first three-dimensional simulation were performed by Klypin \& Shandarin 
(1983), demonstrating the origin of filaments in the dark matter density field. For review see Shandarin \& Zeldovich (1989) where the developments of the original Zeldovich's idea were summarized. The development of perturbations at non-linear stage and structure formation in the medium with barionic gas and non-collisional cold dark matter are investigated by using numerical modelling by many groups (see, for example, Doroshkevich et al (1999), Boily et al (1999), Sneth at al (2001), Boily et al (2002)). For numerical modelling N-body simulations are used, which are very time consuming.

The first stage of large scale structure formation is a collapse of non-collisional dark matter with formation of a pancake and subsequent relaxation with formation of quasiequilibrium structures. This stage can be investigated using approximate model of large scale structure formation considered in works of Bisnovatyi-Kogan (2004) and Bisnovatyi-Kogan \& Tsupko (2005). Form of the collapsing matter have been approximated as rotating homogeneous ellipsoid with two (Bisnovatyi-Kogan 2004) and three (Bisnovatvi-Kogan \& Tsupko 2005) degrees of freedom. Correct description of pressure effects, attained by such approach, and addition of a relaxation permit to get the dynamics of motion without any numerical singularities. In our model a motion along three axes takes place in the gravitational field of a uniform density ellipsoid, with account of the isotropic pressure, represented in an approximate non-differential way. The relaxation leads to a transformation of the kinetic energy of the ordered motion into the kinetic energy of the chaotic motion, and to increase of the effective pressure and thermal energy. All losses are connected with runaway particles. The collapse of the rotating three-axis ellipsoid is approximated by a system of ordinary differential equations, where the relaxation, and the losses of energy, mass and angular momentum are taken into account phenomenologically (Bisnovatyi-Kogan 2004). The system is solved numerically for several parameters, characterizing the configuration.

\section{Equations of motion with dissipation and numerical results}

Equations of motion of rotating dark matter ellipsoid without any dissipation are derived in subsection 3.1, see eq. (3.10), (3.11), (3.12).

In reality there is relaxation in the collisionless system, connected with phase mixing, which is called "violent relaxation" (Lynden-Bell 1967). This relaxation leads to a transformation of the kinetic energy of the ordered motion into the kinetic energy of the chaotic motion and increases effective pressure and thermal energy.

The main transport process is an effective bulk viscosity. Therefore there is a drag force, which is described phenomenologically by adding of the terms

$$
-\frac{\dot{a}}{\tau_{r e l}}, \quad-\frac{\dot{b}}{\tau_{r e l}}, \quad-\frac{\dot{c}}{\tau_{r e l}}
$$

in the right-hand parts of equations of motion.

Dissipation leads to a heat production. The rate of a heat production leading to the growth of the entropy of the spheroid matter was found in Bisnovatyi-Kogan (2004) from equations of motion (3.10), (3.11), (3.12) with account of (3.4), allowing the energy, mass and angular momentum losses.

Here we have scaled the relaxation time $\tau_{r e l}$ by the Jeans characteristic time

$$
\tau_{J}=\frac{2 \pi}{\omega_{J}}=\frac{2 \pi}{\sqrt{4 \pi G \rho}}=2 \pi \sqrt{\frac{a b c}{3 G m}}
$$


with a constant value of $\alpha_{\text {rel }}$

$$
\tau_{r e l}=2 \pi \alpha_{r e l} \sqrt{\frac{a b c}{3 G m}} .
$$

The energy balance relation follows from the dynamic equations in the form

$$
\begin{gathered}
\frac{d U_{t o t}}{d t}=\frac{\partial U_{g}}{\partial m} \frac{d m}{d t}+\frac{\partial U_{r o t}}{\partial m} \frac{d m}{d t}+\frac{\partial U_{r o t}}{\partial M} \frac{d M}{d t}+\frac{\partial E_{t h}}{\partial \mathcal{E}} \frac{d \mathcal{E}}{d t}-2 \frac{U_{k i n}}{\tau_{r e l}}-\frac{U_{k i n}}{m} \frac{d m}{d t}, \\
U_{t o t}=U_{k i n}+U_{g}+U_{r o t}+E_{t h} .
\end{gathered}
$$

The process of relaxation is accompanied also by the energy, mass, and angular momentum losses from the system. We suggest, that these losses take place only in nonstationary phases, so these rates are proportional to the kinetic energy $U_{k i n}$, the characteristic times for mass, angular momentum and energy losses $\tau_{m l}, \tau_{M l}, \tau_{e l}$ are considerably greater then $\tau_{r e l}$. The equations describing different losses may be phenomenologically written as

$$
\frac{d U_{t o t}}{d t}=-\frac{U_{k i n}}{\tau_{m l}}, \quad \frac{d m}{d t}=\frac{m U_{k i n}}{U_{g} \tau_{m l}}, \quad U_{g}<0, \quad \frac{M}{M_{i n}}=\left(\frac{m}{m_{i n}}\right)^{\frac{\alpha_{m l}}{\alpha_{M l}}},
$$

where $m_{i n}$ and $M_{i n}$ are the initial values of corresponding parameters. Scaling all the characteristic times by the Jeans value, we have $\tau_{e l}=\alpha_{e l} \tau_{J}, \quad \tau_{m l}=\alpha_{m l} \tau_{J}, \quad \tau_{M l}=$ $\alpha_{M l} \tau_{J}$, with constant values of $\alpha_{i}(i=e l, m l, M l)$. The function $\mathcal{E}$ determines the entropy of the matter in the spheroid, so that, using (3.9), we have

$$
\frac{d E_{t h}}{d t}+P \frac{d V}{d t}=\frac{1}{(a b c)^{2 / 3}} \frac{d \mathcal{E}}{d t}, E_{t h}=\frac{\mathcal{E}}{(a b c)^{2 / 3}}, V=\frac{4 \pi}{3} a b c, P=\frac{2}{3} \frac{E_{t h}}{V} .
$$

Using (8.5) in (8.4) we obtain the equation for the entropy function $\mathcal{E}$ in presence of different losses in the form

$$
\frac{d \mathcal{E}}{d t}=(a b c)^{2 / 3} U_{k i n}\left[\left(\frac{2}{\tau_{r e l}}-\frac{1}{\tau_{e l}}-\frac{2}{\tau_{m l}}\right)-\frac{U_{r o t}}{U_{g}}\left(\frac{2}{\tau_{M l}}-\frac{1}{\tau_{m l}}\right)+\frac{U_{k i n}}{U_{g} \tau_{m l}}\right] .
$$

The equations, describing approximately the dynamics of the formation of a stationary dark matter object include equations of motion (3.10), (3.11), (3.12) after adding terms with relaxation; energy equation (8.7) with account of (3.5), (3.6), (3.8); and equations (8.5), describing the losses of the energy, mass and angular momentum.

To obtain a numerical solution of equations we write them in non-dimensional variables. Let us introduce the variables

$$
\begin{gathered}
\tilde{t}=\frac{t}{t_{0}}, \tilde{a}=\frac{a}{a_{0}}, \tilde{b}=\frac{b}{a_{0}}, \tilde{c}=\frac{c}{a_{0}}, \tilde{m}=\frac{m}{m_{0}}, \tilde{M}=\frac{M}{M_{0}}, \tilde{\rho}=\frac{\rho}{\rho_{0}}, \\
\tilde{U}=\frac{U}{U_{0}}, \tilde{E}_{t h}=\frac{E_{t h}}{U_{0}}, \tilde{\varepsilon}=\frac{\varepsilon}{\varepsilon_{0}}, \tilde{\tau_{i}}=\frac{\tau_{i}}{t_{0}} .
\end{gathered}
$$

The scaling parameters $t_{0}, a_{0}, m_{0}, M_{0}, \rho_{0}, U_{0}, \Omega_{0}, \varepsilon_{0}$ are connected by the following relations

$$
t_{0}^{2}=\frac{a_{0}^{3}}{G m_{0}}, M_{0}^{2}=G a_{0} m_{0}^{3}, U_{0}=\frac{G m_{0}^{2}}{a_{0}}, \rho_{0}=\frac{m_{0}}{a_{0}^{3}}, \Omega_{0}=\frac{M_{0}}{m_{0} a_{0}^{2}}, \varepsilon_{0}=U_{0} a_{0}^{2} .
$$

$U_{0}$ is used for scaling of all kind of energies. Hereinafter the 'tilde' sign is omitted for simplicity. In non-dimensional variables we have $m=\frac{4 \pi}{3} \rho a b c, M=\frac{m}{5} \Omega\left(a^{2}+b^{2}\right), \tau_{i}=$ $2 \pi \alpha_{i} \sqrt{\frac{a b c}{3 m}}$. 
Taking into account violent relaxation, total energy, mass and angular momentum losses, the dynamics of the system is described by the following non-dimensional system of equations

$$
\begin{gathered}
\ddot{a}=-\frac{\dot{a}}{m} \frac{d m}{d t}-\frac{3 m}{2} a \int_{0}^{\infty} \frac{d u}{\left(a^{2}+u\right) \Delta}+\frac{10}{3 m} \frac{1}{a} \frac{\varepsilon}{(a b c)^{2 / 3}}+\frac{25 M^{2}}{m^{2}} \frac{a}{\left(a^{2}+b^{2}\right)^{2}}-\frac{\dot{a}}{\tau_{r e l}}, \\
\ddot{b}=-\frac{\dot{b}}{m} \frac{d m}{d t}-\frac{3 m}{2} b \int_{0}^{\infty} \frac{d u}{\left(b^{2}+u\right) \Delta}+\frac{10}{3 m} \frac{1}{b} \frac{\varepsilon}{(a b c)^{2 / 3}}+\frac{25 M^{2}}{m^{2}} \frac{b}{\left(a^{2}+b^{2}\right)^{2}}-\frac{\dot{b}}{\tau_{r e l}}, \\
\ddot{c}=-\frac{\dot{c}}{m} \frac{d m}{d t}-\frac{3 m}{2} c \int_{0}^{\infty} \frac{d u}{\left(c^{2}+u\right) \Delta}+\frac{10}{3 m} \frac{1}{c} \frac{\varepsilon}{(a b c)^{2 / 3}}-\frac{\dot{c}}{\tau_{r e l}} \\
\dot{\varepsilon}=(a b c)^{2 / 3} U_{k i n}\left[\left(\frac{2}{\tau_{r e l}}-\frac{1}{\tau_{e l}}-\frac{2}{\tau_{m l}}\right)-\frac{U_{r o t}}{U_{g}}\left(\frac{2}{\tau_{M l}}-\frac{1}{\tau_{m l}}\right)+\frac{U_{k i n}}{U_{g} \tau_{m l}}\right] \\
\dot{m}=-\frac{1}{3 \tau_{m l}}\left(\dot{a}^{2}+\dot{b}^{2}+\dot{c}^{2}\right) /\left(\int_{0}^{\infty} \frac{d u}{\Delta}\right), \frac{M}{M_{i n}}=\left(\frac{m}{m_{i n}}\right)^{\frac{\tau_{m l}}{\tau_{M l}}}, \Delta^{2}=\left(a^{2}+u\right)\left(b^{2}+u\right)\left(c^{2}+u\right)
\end{gathered}
$$

This system is solved numerically for several initial parameters. The case of spheroid $(a=b \neq c)$ is considered in the paper of Bisnovatvi-Kogan (2004), the case of ellipsoid in the paper of Bisnovatyi-Kogan \& Tsupko (2005). For investigation of the dynamical behavior we start the simulation from spherical body of unit mass $\left(m_{i n}=1\right)$, zero or small entropy $\varepsilon \ll 1$. We also specify the parameters, characterizing different dissipations. In all calculations with relaxation we use the following values: $\alpha_{r e l}=3, \alpha_{e l}=\alpha_{m l}=\alpha_{M l}=15$. We set non-zero initial velocities. Because of the rotation around $c$-axis, the initial sphere transforms to spheroid during the collapse, if initial parameters for axes $a$ and $b$ are exactly equal $\left(a_{i n}=b_{i n}, \dot{a}_{i n}=\dot{b}_{i n}\right)$. Note that during the motion spheroids may be not only oblate but prolate too (Bisnovatyi-Kogan 2004). To study an appearance of 3-axis figures the initial parameters for axes $a$ and $b$ had been slightly different in all variants of calculations.

In the first variant $(\operatorname{Fig} 8)$ there is a large initial angular momentum $M_{i n}=0.5$. The field of velocities is slightly perturbed by increasing $\dot{b}$ in comparison with $\dot{a}$. Initially we observe the collapse and the formation of the pancake. During the motion the difference $(a-b)$ increases due to development of a secular instability, and we obtain the transformation of the Maclaurin spheroid into the Jacobi ellipsoid in the dynamics. Because of the relaxation, the oscillating motion damps, and the configuration reaches the equilibrium state of the 3-axis ellipsoid. The corresponding behaviors of total energy $U_{t o t}$, entropy function $\varepsilon$ and mass $m$ are represented in Figs 9 , 10, 11, We see that the main changes of these parameters take place at the stages of first few oscillations: the entropy function $\varepsilon$ increases, the mass $m$ and the total energy $U_{t o t}$ decreases. Then these parameters reaches the equilibrium values.

In the second variant of calculations (Fig 12) we set a small initial angular momentum $M_{i n}=0.1$. After the collapse, there are temporary oblate and prolate spheroid appearance during relaxation. There is no secular instability, and the system reaches the equilibrium oblate spheroid of Maclaurin. Here we have an additional instability, there 


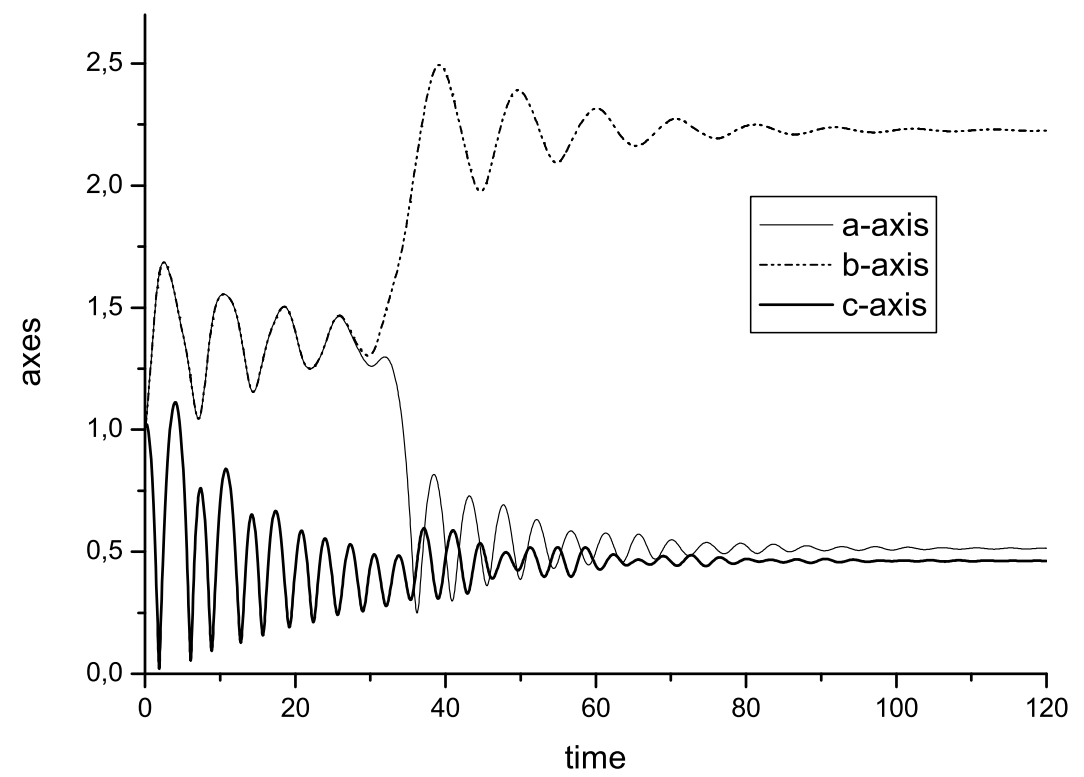

FIgURE 8. Development of an instability at large angular momentum, and formation of a stationary triaxial figure.

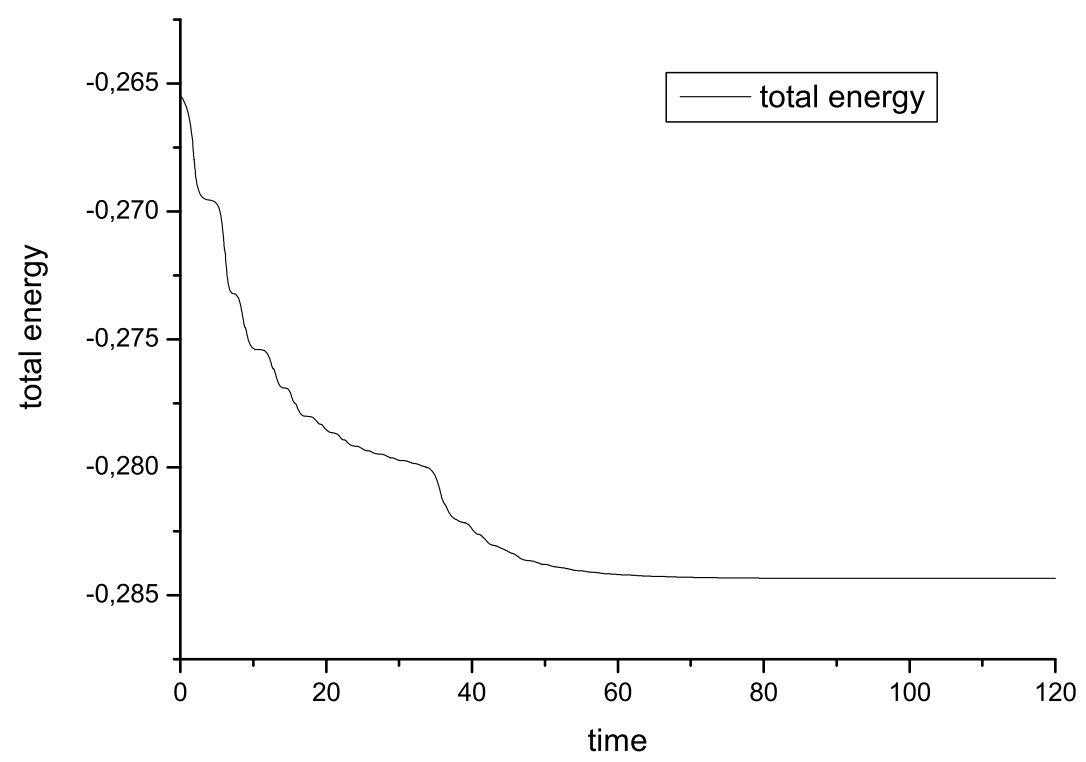

Figure 9. The total energy evolution for the case considered on Fig 8 


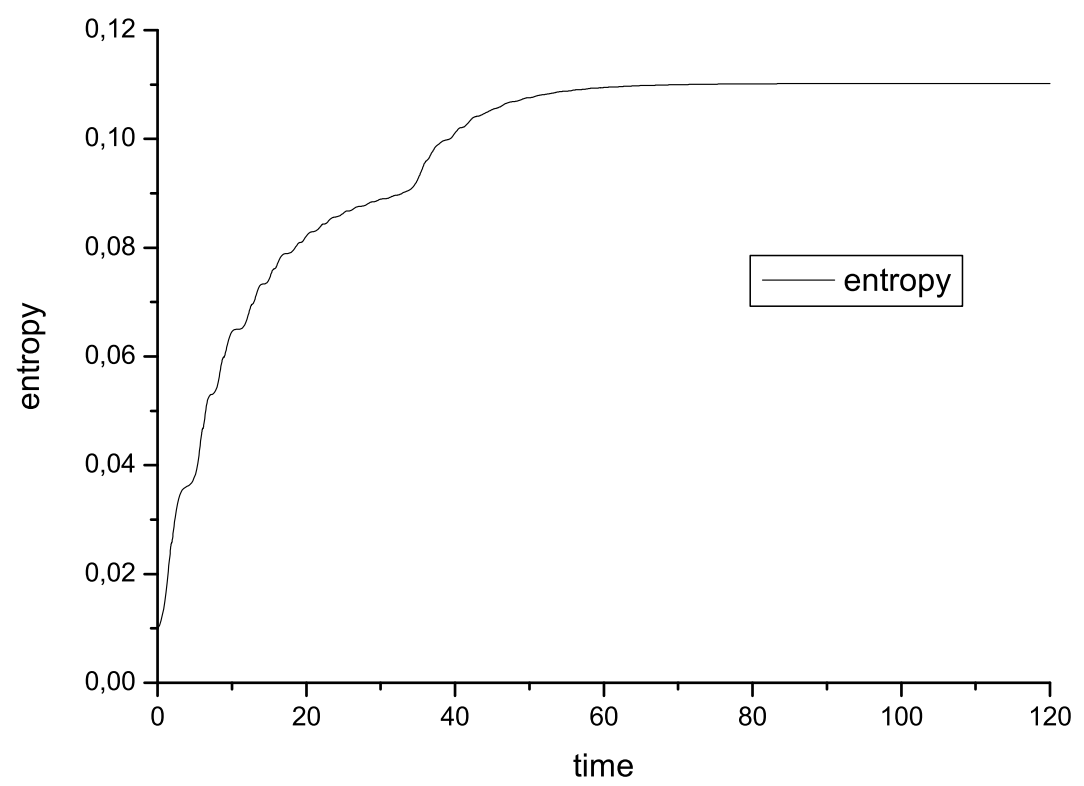

FiguRE 10. The entropy function evolution for the case considered on Fig 8

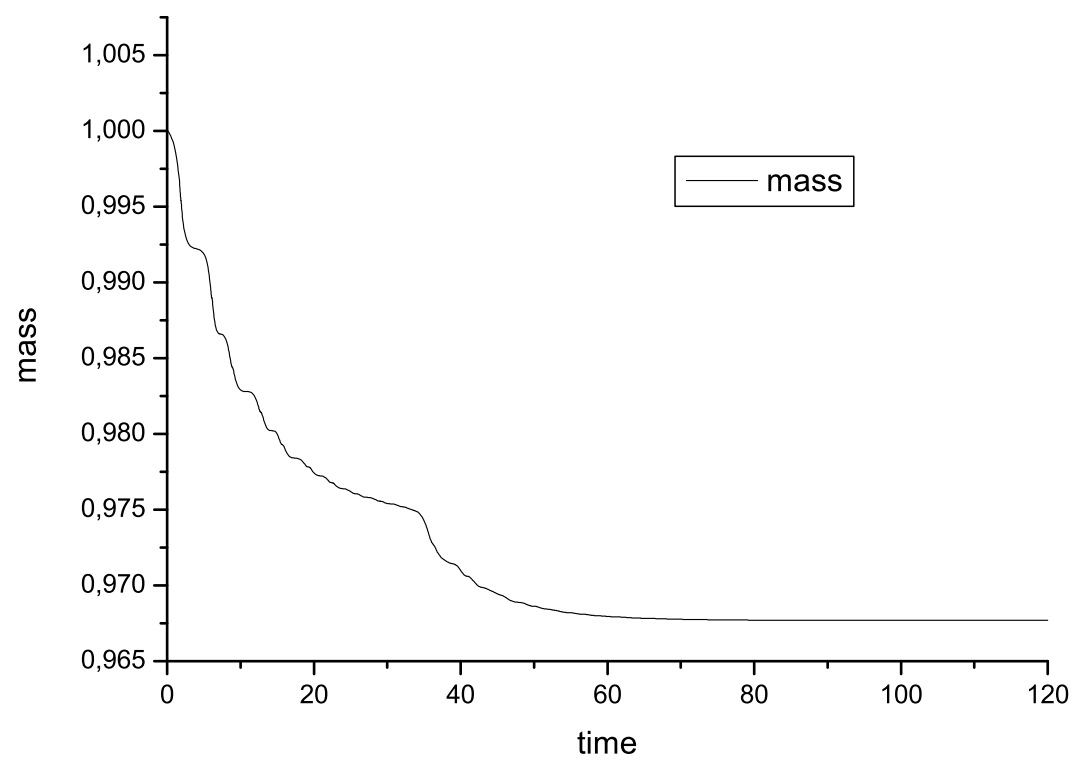

Figure 11. The mass evolution for the case considered on Fig 8 


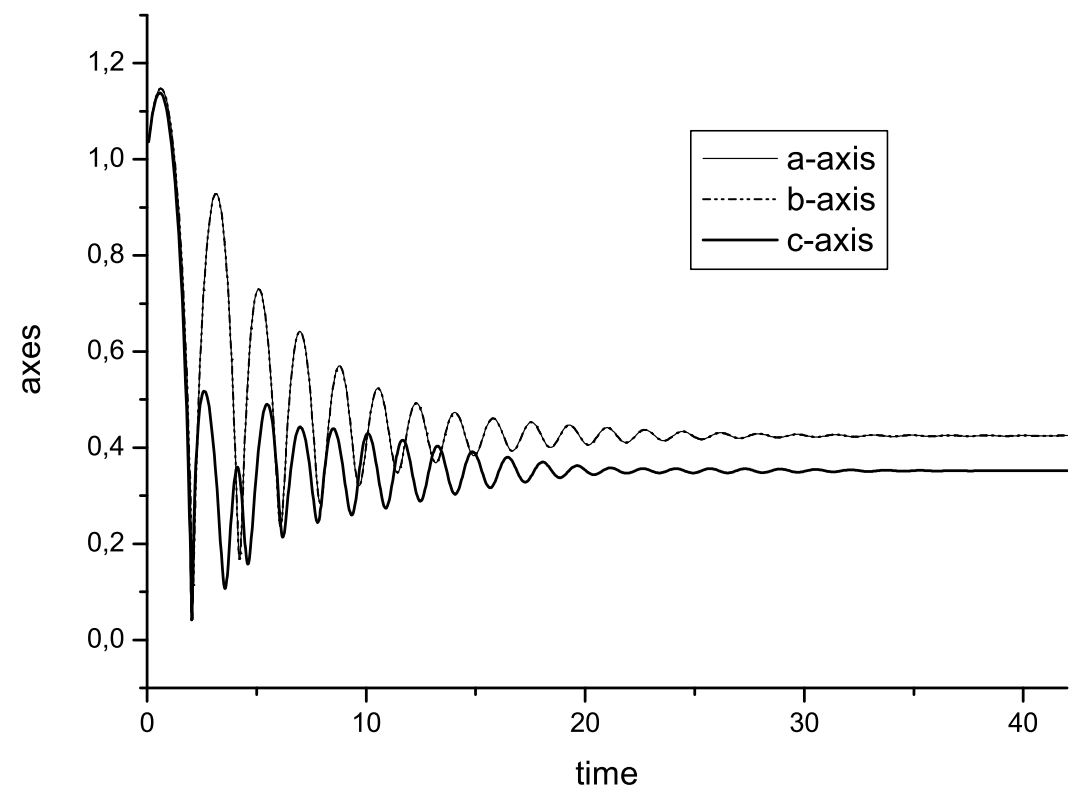

FIGURE 12. The relaxation to the spheroid at small angular momentum.

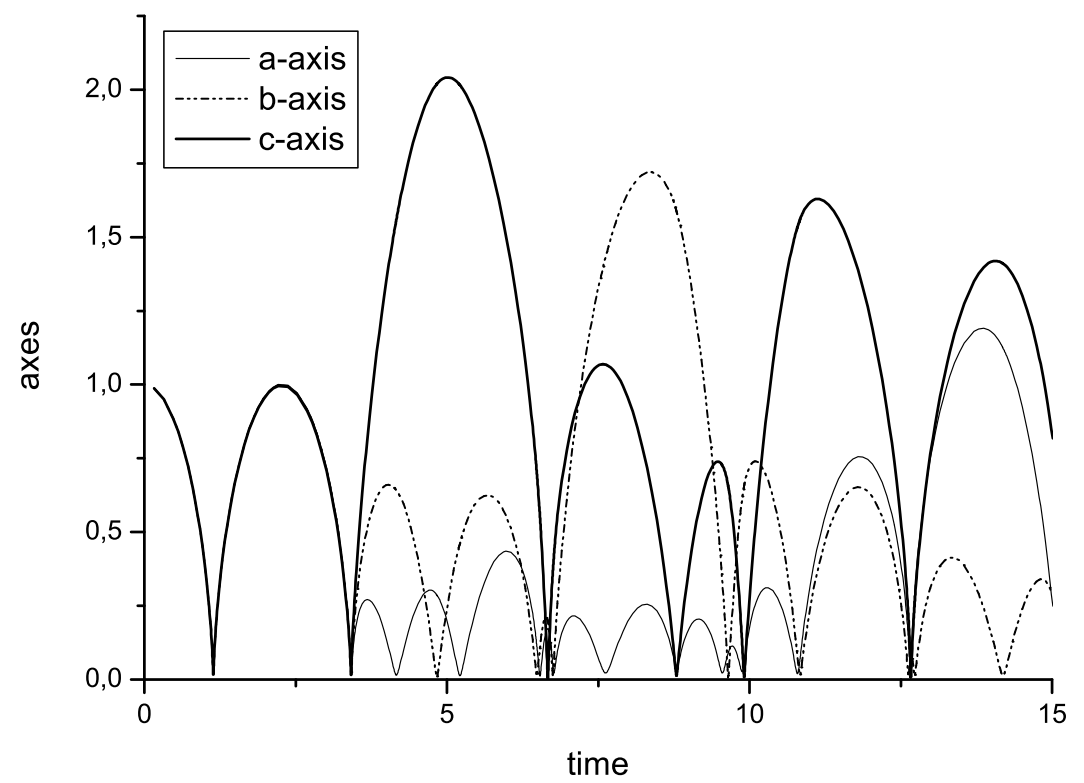

FIGURE 13. Radial instability development in the non-rotating body, without dissipation. 


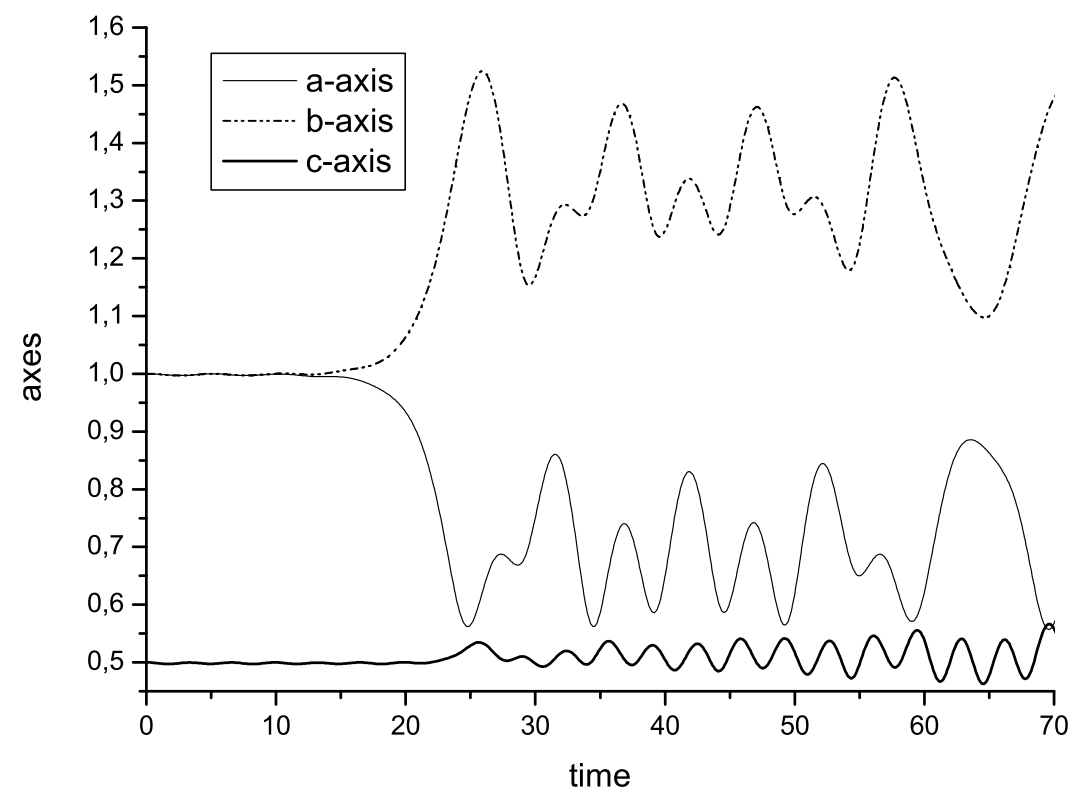

FIGURE 14. Development of the secular instability in a rapidly rotating body, without dissipation.

is increasing of the difference $(a-b)$ at early stages of motion. However this difference remains too small for visualizing in Figure. This is the instability characteristic to the system with purely radial trajectories (Antonov 1973; Fridman \& Polvachenko 1985). This instability takes place in all variants of calculations, but in most cases it is very small and always disappears during the relaxation and formation of the equilibrium figure. In the cases of large angular momentum this instability does not reveal because the difference $(a-b)$ quickly increases as a result of the secular instability.

For the illustration of the radial instability we consider initial sphere without rotation $\left(M_{i n}=0\right)$ and without any relaxation process. Only three equations (8.8)-8.10) with $\tau_{r e l}=\infty$ have been used, at constant $m=1, M=0$ and $\varepsilon$. We have set small initial differences between axes $a, b$ and $c$, and have obtained an unstable behavior evidently visible in Fig 13 ,

For numerical investigation of the secular instability we consider equations of motion without dissipation, and take the equilibrium spheroids with small perturbation as the initial configuration. It is easy to obtain equilibrium parameters of the spheroid from equations of motion with zero accelerations. The initial equilibrium configuration of the Maclaurin spheroid was slightly perturbed by increasing $b$. At small angular momentum we obtain the conservation of the initial configuration. There are small oscillations around the equilibrium spheroid, during which the difference $(a-b)$ changes sign periodically. At large angular momentum we obtain the development of the secular instability, and, after several low-amplitude oscillations, the Maclaurin spheroid, is transformed into the Jacobi ellipsoid (see Fig 14 where the variant with large angular momentum is represented). Because of absence of relaxation, the system does not reach equilibrium configuration and oscillations continue. If we include relaxation in this variant, we should observe the final 
equilibrium state of ellipsoid. Our calculations allow to find approximately numerically the point of the onset of the secular instability of the compressible Maclaurin spheroid.

\section{Equilibrium configurations and stability}

Equilibrium of the uniformly rotating figure (spheroid or ellipsoid) is found from the equations (8.8)- (8.10) with zero time derivatives:

$$
\begin{gathered}
0=-\frac{3 m}{2} a \int_{0}^{\infty} \frac{d u}{\left(a^{2}+u\right) \Delta}+\frac{10}{3 m} \frac{1}{a} \frac{\varepsilon}{(a b c)^{2 / 3}}+\frac{25 M^{2}}{m^{2}} \frac{a}{\left(a^{2}+b^{2}\right)^{2}}, \\
0=-\frac{3 m}{2} b \int_{0}^{\infty} \frac{d u}{\left(b^{2}+u\right) \Delta}+\frac{10}{3 m} \frac{1}{b} \frac{\varepsilon}{(a b c)^{2 / 3}}+\frac{25 M^{2}}{m^{2}} \frac{b}{\left(a^{2}+b^{2}\right)^{2}}, \\
0=-\frac{3 m}{2} c \int_{0}^{\infty} \frac{d u}{\left(c^{2}+u\right) \Delta}+\frac{10}{3 m} \frac{1}{c} \frac{\varepsilon}{(a b c)^{2 / 3}} .
\end{gathered}
$$

Introducing variables

and integrals

$$
x=\frac{u}{a^{2}}, k=\frac{c}{a}, k_{1}=\frac{b}{a}, j=\frac{M}{m}, \epsilon=\frac{\varepsilon}{m},
$$

$$
\begin{gathered}
I_{1}\left(k, k_{1}\right)=\int_{0}^{\infty} \frac{d x}{(x+1)^{3 / 2} \sqrt{\left(k_{1}^{2}+x\right)\left(k^{2}+x\right)}}, \quad I_{2}\left(k, k_{1}\right)=\int_{0}^{\infty} \frac{d x}{\left(x+k_{1}^{2}\right)^{3 / 2} \sqrt{(1+x)\left(k^{2}+x\right)}}, \\
I_{3}\left(k, k_{1}\right)=\int_{0}^{\infty} \frac{d x}{\left(x+k^{2}\right)^{3 / 2} \sqrt{(1+x)\left(k_{1}^{2}+x\right)}}
\end{gathered}
$$

we write the equations (9.1)-(9.3), using (8.12) in the form

$$
\begin{gathered}
0=-\frac{3 m}{2} a I_{1}\left(k, k_{1}\right)+\frac{10}{3} \frac{\epsilon}{\left(k k_{1}\right)^{2 / 3}}+\frac{25 j^{2}}{\left(1+k_{1}^{2}\right)^{2}}, \\
0=-\frac{3 m}{2} a k_{1} I_{2}\left(k, k_{1}\right)+\frac{10}{3} \frac{\epsilon}{k_{1}\left(k k_{1}\right)^{2 / 3}}+\frac{25 j^{2} k_{1}}{\left(1+k_{1}^{2}\right)^{2}}, \\
0=-\frac{3 m}{2} a k I_{3}\left(k, k_{1}\right)+\frac{10}{3} \frac{\epsilon}{k\left(k k_{1}\right)^{2 / 3}} .
\end{gathered}
$$

Excluding $m$, we obtain

$$
0=-\frac{1}{k^{2}} \frac{I_{1}\left(k, k_{1}\right)}{I_{3}\left(k, k_{1}\right)}+1+\frac{15 j^{2}}{2 \epsilon} \frac{\left(k k_{1}\right)^{2 / 3}}{\left(1+k_{1}^{2}\right)^{2}}, \quad 0=-\frac{k_{1}^{2}}{k^{2}} \frac{I_{2}\left(k, k_{1}\right)}{I_{3}\left(k, k_{1}\right)}+1+k_{1}^{2} \frac{15 j^{2}}{2 \epsilon} \frac{\left(k k_{1}\right)^{2 / 3}}{\left(1+k_{1}^{2}\right)^{2}} .
$$

At $a=b, k_{1}=1$ equations (9.9) are identical, and determine the equilibrium of Maclaurin spheroid, see Bisnovatyi-Kogan (2004). For Jacobi ellipsoids with $a \neq b \neq c$, we obtain the following relation between $k$ and $k_{1}$

$$
F\left(k, k_{1}\right)=1-\frac{1}{k_{1}^{2}}+\frac{1}{k^{2}} \frac{I_{2}\left(k, k_{1}\right)}{I_{3}\left(k, k_{1}\right)}-\frac{1}{k^{2}} \frac{I_{1}\left(k, k_{1}\right)}{I_{3}\left(k, k_{1}\right)}=0 .
$$


This equation has a trivial solution $k_{1}=1$ at all $k$, corresponding to the Maclairin spheroid. Let us find the bifurcation point of the equation (9.10), at which nontrivial solutions appear. While $k_{1}=1$ is always a root of the equation (9.10), we may write $F\left(k, k_{1}\right)=\left(k_{1}-1\right) f\left(k, k_{1}\right)$. Additional root of equation $F\left(k, k_{1}\right)=0$ appears, when the root of the equation $f\left(k, k_{1}\right)=0$ appears at $k_{1}=1$. The root of the zero derivative equation $F_{k_{1}}^{\prime}\left(k, k_{1}\right)=f\left(k, k_{1}\right)+\left(k_{1}-1\right) f_{k_{1}}^{\prime}\left(k, k_{1}\right)=0$ at $k_{1}=1$ coincides with the root of the equation $f(k, 1)=0 \mathbb{H}$, therefore the value of $k$ at the bifurcation point is determined by the equation

$$
\left.\frac{\partial F\left(k, k_{1}\right)}{\partial k_{1}}\right|_{k_{1}=1}=0
$$

Using (9.5) and (9.10), this equation is written in the form

$$
\left.2 k^{2} I_{3}\left(k, k_{1}\right)\right|_{k_{1}=1}+\left.\frac{\partial I_{2}\left(k, k_{1}\right)}{\partial k_{1}}\right|_{k_{1}=1}-\left.\frac{\partial I_{1}\left(k, k_{1}\right)}{\partial k_{1}}\right|_{k_{1}=1}=0
$$

At $k_{1}=1$ we have the analytic expressions

$$
\left.\frac{\partial I_{1}\left(k, k_{1}\right)}{\partial k_{1}}\right|_{k_{1}=1}=-I_{0},\left.\quad \frac{\partial I_{2}\left(k, k_{1}\right)}{\partial k_{1}}\right|_{k_{1}=1}=-3 I_{0}, \quad I_{0}=\int_{0}^{\infty} \frac{d x}{(1+x)^{3} \sqrt{k^{2}+x}},
$$

so that the equation (9.12) is reduced to

$$
k^{2} I_{3}=I_{0}, \quad \text { where } I_{3}=I_{3}(k, 1), \quad \text { and } I_{2}=I_{2}(k, 1)=I_{1}=I_{1}(k, 1) .
$$

Taking analytically the integrals $I_{0}, I_{1}=I_{2}$ and $I_{3}$ we have

$$
\begin{gathered}
\left(1-k^{2}\right) I_{0}=\frac{3}{4} I_{2}-\frac{k}{2}, \quad I_{3}=\frac{2}{k\left(1-k^{2}\right)}\left(1-\frac{k \arccos k}{\sqrt{1-k^{2}}}\right), \\
I_{1}=I_{2}=-\frac{1}{k\left(1-k^{2}\right)}\left(k^{2}-\frac{k \arccos k}{\sqrt{1-k^{2}}}\right) .
\end{gathered}
$$

Using (9.15) in (9.14) we obtain the equation

$$
\frac{\arccos k}{\sqrt{1-k^{2}}}=\frac{k\left(13-10 k^{2}\right)}{3+8 k^{2}-8 k^{4}},
$$

which solution $k=0.582724$ determines the bifurcation point at the sequence of the Maclaurin spheroids. For the uniform spheroid the position of this point does not depend on the adiabatic index of the matter.

Above we have obtained the bifurcation point on the equilibrium curve of the Maclaurin spheroids using only the equilibrium relations for the Jacobi ellipsoids. The usual way for investigation of stability is to solve linearized equations of motion and to find the eigenfrequencies.

Non-dimensional equations of motion without relaxation are

$$
\begin{aligned}
& \ddot{a}=-\frac{3 m}{2} a \int_{0}^{\infty} \frac{d u}{\left(a^{2}+u\right) \Delta}+\frac{10}{3 m} \frac{1}{a} \frac{\varepsilon}{(a b c)^{2 / 3}}+\frac{25 M^{2}}{m^{2}} \frac{a}{\left(a^{2}+b^{2}\right)^{2}}, \\
& \ddot{b}=-\frac{3 m}{2} b \int_{0}^{\infty} \frac{d u}{\left(b^{2}+u\right) \Delta}+\frac{10}{3 m} \frac{1}{b} \frac{\varepsilon}{(a b c)^{2 / 3}}+\frac{25 M^{2}}{m^{2}} \frac{b}{\left(a^{2}+b^{2}\right)^{2}},
\end{aligned}
$$

$\dagger$ We are grateful to A.I.Neishtadt for useful discussion of this point 


$$
\ddot{c}=-\frac{3 m}{2} c \int_{0}^{\infty} \frac{d u}{\left(c^{2}+u\right) \Delta}+\frac{10}{3 m} \frac{1}{c} \frac{\varepsilon}{(a b c)^{2 / 3}}, \quad \Delta^{2}=\left(a^{2}+u\right)\left(b^{2}+u\right)\left(c^{2}+u\right) .
$$

At given $a, b, c$ the equilibrium values of $\varepsilon M$ (the equilibrium configuration of ellipsoid) are obtained using formulae:

$$
\begin{gathered}
\varepsilon=\frac{9}{20} m^{2} c^{2}(a b c)^{2 / 3} \int_{0}^{\infty} \frac{d u}{\left(c^{2}+u\right) \Delta}, \\
\frac{25 M^{2}}{m^{2}} \frac{a^{2}}{\left(a^{2}+b^{2}\right)^{2}}=\frac{3 m}{2} a^{2} \int_{0}^{\infty} \frac{d u}{\left(a^{2}+u\right) \Delta}-\frac{3 m}{2} c^{2} \int_{0}^{\infty} \frac{d u}{\left(c^{2}+u\right) \Delta} .
\end{gathered}
$$

We linearize equations (9.17) and (9.18) around the equilibrium configuration of the Maclaurin spheroid $(a=b, k=c / a)$. Subtracting (9.17) and (9.18) with using equilibrium values (9.20) and (9.21), we obtain

$$
\delta(\ddot{a}-\ddot{b})=3 \frac{m}{a^{3}}\left[-k^{2} \int_{0}^{\infty} \frac{d u}{(1+u)\left(k^{2}+u\right)^{3 / 2}}+\int_{0}^{\infty} \frac{d u}{(1+u)^{3}\left(k^{2}+u\right)^{1 / 2}}\right] \delta(a-b) .
$$

The $\varepsilon, U_{t o t}, m$ and $M$ losses are quadratic to perturbations, so these values remain constant in linear approximation.

Taking $\delta(a-b) \sim \exp (-i \omega t)$ we come to the characteristic equation

$$
\omega^{2}=3 \frac{m}{a^{3}}\left[k^{2} \int_{0}^{\infty} \frac{d u}{(1+u)\left(k^{2}+u\right)^{3 / 2}}-\int_{0}^{\infty} \frac{d u}{(1+u)^{3}\left(k^{2}+u\right)^{1 / 2}}\right] .
$$

These integrals are expressed in analytical functions:

$\omega^{2}=\frac{3 m}{a^{3}}\left[k^{2}\left(\frac{2}{k\left(1-k^{2}\right)}-\frac{2 \arccos k}{\left(1-k^{2}\right)^{3 / 2}}\right)-\left(\frac{3 \arccos k}{4\left(1-k^{2}\right)^{5 / 2}}-\frac{3 k}{4\left(1-k^{2}\right)^{2}}-\frac{k}{2\left(1-k^{2}\right)}\right)\right]$.

For the border of stability $\omega^{2}=0$, we obtain the equation again the equation (9.16).

The spheroid loses its stability for the transformation into three-axial ellipsoid, at the bifurcation point $k=0.58272, e=\sqrt{1-c^{2} / a^{2}}=0.81267$. Our approximate equilibrium equations, even without dissipation, describe the uniformly rotating ellipsoids (spheroids), which are not connected by adiabatic relations, and contain a "hidden" nonconservation of the local angular momentum, which preserves the uniformity. Therefore, in presence of this "hidden" non-conservation, the loss of stability takes place exactly in the bifurcation point. The loss of stability is not connected with the relaxation and follows from our equations even in the case when all global integrals remain constant. In the exact approach the instability in this point happens only at the direct presence of dissipative terms (Chandrasekhar 1969). The pure adiabatic spheroidal system preserves stability until $e=0.952887$, where it becomes unstable via a vibrational mode.

According to the hypothesis of Ostriker \& Peebles (1973), the stability of an isolated axially symmetric system is determined by the ratio $U_{\text {rot }} /\left|U_{g}\right|$. They determined from numerical experiments the critical value for various configuration as $0.14 \pm 0.03$. It was found in Lai, Rasio \& Shapiro (1993) and Shapiro (2004) that compressible spheroids become secularly unstable to triaxial deformations at the bifurcation point, where $U_{\text {rot }} /\left|U_{g}\right|=$ 0.1375 , independent of the adiabatic index $n, \gamma=1+\frac{1}{n}$. Our formula gives exactly the same result, which is also confirmed by our numerical simulations. 


\section{Emission of very long gravitational waves and gravitational lensing by gravitational waves}

Gravitational radiation is produced during the collapse of the non-spherical body. Gravitational radiation during a formation of a pancake was estimated by Thuan and Ostriker (1974), and was improved by Novikov (1975), who took into account the most important stage of the radiation during a bounce. The formula for the estimation of the total energy emitted during the collapse and bounce is

$$
U_{G W} \approx 0.01\left(\frac{r_{g}}{a_{e q}}\right)^{7 / 2}\left(\frac{a_{e q}}{c_{\min }}\right) M c^{2} .
$$

Here $a_{e q}$ is the equilibrium equatorial radius of the pancake, and $c_{\min }$ is its minimal half-thickness during the bounce, $r_{g}=2 G M / c^{2}$ is the Schwarzschild gravitational radius of the body. From our calculations we have $a_{e q} / c_{m i n} \leqslant 100$. The value of $r_{g} / a_{e q}$ we estimate using the observed average velocity of a galaxy in the rich cluster $v_{g} \sim 3000$ $\mathrm{km} / \mathrm{s}$, and taking

$$
\frac{r_{g}}{a_{e q}} \sim\left(\frac{v_{g}}{c}\right)^{2} \approx 10^{-4}
$$

Than the energy carried away by the gravitational wave (GW) may be estimated as $U_{G W} \approx 10^{-14} M c^{2}$. If all dark matter had passed through the stage of a pancake formation, than very long GW with a wavelength of the order of the size of the galactic cluster have an average energy density in the universe

$$
\varepsilon_{G W} \approx 10^{-14} \rho_{d m} c^{2} \approx 3 \cdot 10^{-23} \mathrm{erg} / \mathrm{cm}^{3} .
$$

Here we have used for estimation the average dark matter density $\rho_{d m}=3 \cdot 10^{-30} \mathrm{~g} / \mathrm{cm}^{3}$. The average strength $E_{G W}$ of the very long GW may be estimated, taking the relation (Landau \& Lifshitz 1993)

$$
\varepsilon_{G W}=\frac{c^{2}}{16 \pi G} \dot{h}^{2}
$$

where $h$ is metric tensor perturbation (non-dimensional), connected with GW, we consider only the scalar, having in mind the averaged value of this perturbation. Taking into account $\dot{h}=\omega h=2 \pi c h / \lambda, \lambda \sim 10 \mathrm{Mpc}$ is the wavelength of GW of the order of the size of the cluster. From the comparison of (10.3), (10.4) we obtain the averaged metric perturbation due to very long GW in the form (Bisnovatyi-Kogan 2004)

$$
\bar{h}=\frac{2 \lambda}{c^{2}}\left(\frac{G \varepsilon_{G W}}{\pi}\right)^{1 / 2} \approx 6 \cdot 10^{-11}
$$

for the values of $\lambda$ and $\varepsilon_{G W}$, mentioned above. Incidently we may expect 10 times larger amplitude of the GW, than the averaged over the volume value.

According to general relativity, any gravitational field can change trajectory of photons or, in other words, deflect light rays. Hence the gravitational field may act as a gravitational lens.

Gravitational lensing by gravitational waves in different cases was considered by many authors (see Braginsky et al (1990), Faraoni (1992), Damour and Esposito-Farèse (1998) and references therein). It was found that the deflection angle vanishes for any localized gravitational wave packet because of transversality of gravitational waves (Damour and Esposito-Farèse 1998). Thus if the photon passes through the finite gravitational wave pulse its deflection due to this wave is equal to zero. In Bisnovatyi-Kogan and Tsupko (2008b) we notice that the displacement between trajectories of the photon before and after passing the 


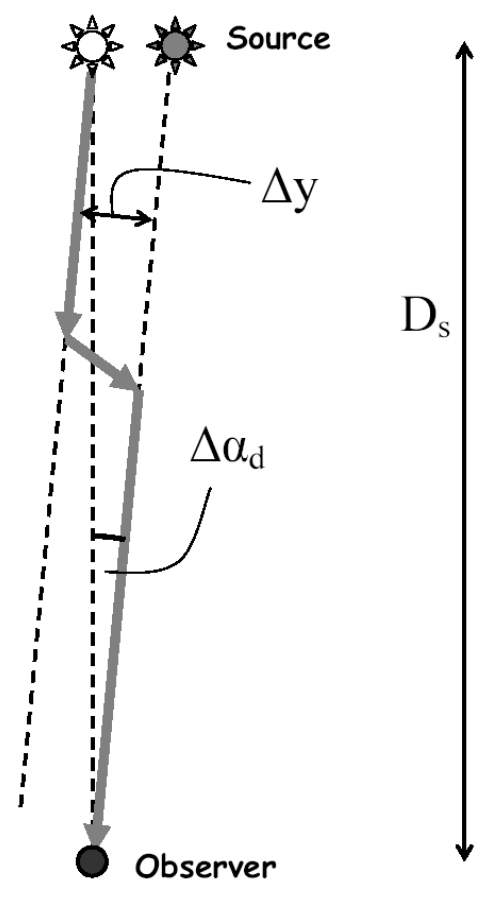

Figure 15. The observational effect of the displacement in trajectory of the photon.

wave may occur. On the basis of this result we obtain an approximate formula for the estimation of observational effects.

We confirm analytically vanishing of deflection angle for plane wave pulses. However, we have found that the gravitational wave $(\mathrm{GW})$ changes the photon propagation in another way, simply shifting its whole trajectory after passing through the GW (see fig. 15). This displacement is found analytically for the photon passing through the plane GW. Displacement takes place mainly in the case of isolated wave pulses, which have a form similar to the top part of the sinusoid or when it has the non-symmetrical top and bottom parts of wave profile, and may, in principal, vanish for the periodic wave of a long duration.

Directions of photons passing through the gravitational wave packet does not change, therefore any focusing of rays does not occur in this case. Thus the displacement in trajectories does not lead to any magnification effect. But the displacement leads to change of the angular position of object for distant observer. The change of the angular position due to passing of the light ray through the gravitational wave pulse $\Delta \alpha_{d}$ is as (see fig. 15)

$$
\Delta \alpha_{d}=\frac{\Delta y}{D_{s}} \simeq \frac{h \delta}{D_{s}}
$$

where $h$ is the amplitude of the GW pulse, $\delta$ is its thickness and $D_{s}$ is a distance between the source and the observer.

Let us estimate the change of the angular position for the GW pulses produces during formation of large scale structure of the Universe in dark matter using the following parameters: $h=10^{-11}, \delta=M p c, D_{s}=100 M p c$. Than we obtain

$$
\Delta \alpha_{d} \simeq 2 \cdot 10^{-8} \text { arcsec. }
$$


Another possible manifestation of gravitational waves is influence of them on the polarization of cosmic microwave background (Polnarev 1985). It is known that primordial GWs lead to formation of B-mode polarization (Seliak \& Zaldarriaga 1997), see also textbook of Gorbunov \& Rubakov (2011). This effect is now hunting by different observational projects, e.g. see BICEP2 (http://bicepkeck.org/). We suppose that B-mode polarization formation can be explained not only by primordial gravitational waves but also by gravitational waves appeared in the epoch of the formation of the large scale structure in the universe. The main distinguishing feature of gravitational waves formed during large scale structure formation is anisotropy in direction to rich clusters of galaxies.

\section{Conclusions}

We present here the approximate way for investigation of collapse and dynamic stability based on the model of compressible homogeneous 3-axis ellipsoids. Investigation of dynamics is reduced to numerical solution of system of ordinary differential equations of semi-axes time evolution. In the presence of dissipation processes there are also equations for mass, angular momentum, energy, and entropy evolution. Derivation of equations of motion have been performed by variation of the Lagrange function of the ellipsoid. Different equations of state can be easily considered in this model. Right-hand sides of equations have analytical form for spheroid case and can be written in terms of elliptical integrals for 3-axis ellipsoid case. Equilibrium configurations can be also investigated by taking zero accelerations in all equations. We apply this method for investigation of dynamics of non-spherical stars and dark matter large scale structure formation.

Dynamic stability of non-spherical stars was investigated. We have solved the equations that describe, in a simplified way, the Newtonian dynamics of a self-gravitating non-rotating spheroidal body after loss of stability. We have found that contraction to a singularity occurs only in a pure spherical collapse, and deviations from spherical symmetry stop the contraction through the stabilizing action of non-linear non-spherical oscillations. A real collapse occurs after damping of the oscillations because of energy losses, shock wave formation or viscosity.

We have investigated the dynamics of 3-axis dark matter ellipsoid. Equations of motion for axes of uniform compressible ellipsoid have been obtained by variation of the Lagrange function, in which the violent relaxation and losses of a matter, energy and angular momentum have been included phenomenologically.

The system was solved numerically, until the formation of stationary rotating figures in presence of the relaxation. For lower angular momentum $M$ we have a formation of a compressed spheroid, while at larger $M$ we follow the development of three-axial instability and formation of three-axial ellipsoid. The instability in this approximation happens at the bifurcation point of the sequence of Maclaurin spheroids, where Jacobi ellipsoidal system starts.

The bifurcation point coinciding with the point of loss of stability is found analytically in the form of a simple formula, by static and dynamic approaches. Numerical and analytical considerations give identical results. Development of instability, connected with radial orbits, is obtained for slowly rotating collapsing bodies.

The weak but very long gravitational waves (GW) emitted mainly on the stages of the collapse and pancake formation form a long wave GW background. The existence of such a long GW in the space between the source and the observer may be registered as an action of the gravitational lens. Effect of lensing can be due to displacement of the 
whole trajectory if light ray is passing through the GW pulse. The displacement leads to change of the angular position of object for distant observer.

The challenging idea is explanation of B-mode polarization formation not only by primordial gravitational waves but also by gravitational waves appeared in the epoch of the formation of the large scale structure in the universe.

\section{Acknowledgements}

The work of GSBK and OYuT was partly supported by the Russian Science Support Foundation, Russian Basic Research Foundation Grant No. 14-02-00728 and the Russian Federation President Grant for Support of Leading Scientific Schools, Grant No. NSh261.2014 .2

The work of GSBK was also partially supported by the Russian Foundation for Basic Research Grant No. OFI-M 14-29-06045.

\section{REFERENCES}

Antonov V.A., 1973, The Dynamics of Galaxies and Stellar Clusters. Nauka, Alma-Ata (in Russian)

Ardeljan N.V., Bisnovatyi-Kogan G.S., Moiseenko S.G., 2005, Magnetorotational supernovae, MNRAS, 359, 333-344

Bisnovatyi-Kogan G.S., 1989, Physical problems in the theory of stellar evolution. Nauka, Moscow (in Russian). (English translation: Stellar Physics, Vol. 1,2. Springer, 2001)

Bisnovatyi-Kogan G.S., 2004, A simplified model of the formation of structures in dark matter and a background of very long gravitational waves, MNRAS 347, 163-172

Bisnovatyi-Kogan G.S. and Tsupko O.Yu., 2005, Approximate dynamics of dark matter ellipsoids, MNRAS 364, 833-842

Bisnovatyi-Kogan G.S. and Tsupko O.Yu., 2008a, Dynamic stabilization of non-spherical bodies against unlimited collapse, MNRAS 386, 1398-1403

Bisnovatyi-Kogan G.S. and Tsupko O.Yu., 2008b, Gravitational lensing by gravitational waves, Gravitation and Cosmology, V.14, N.3, 226-229.

Boily C.M., Clarke C.J., Murray S.D. 1999, Collapse and evolution of flattened star clusters, Monthly Notice of the Royal Astronomical Society, 302, 399.

Boily C.M., Athanassoula E., Kroupa P., 2002, Scaling up tides in numerical models of galaxy and halo formation, Monthly Notice of the Royal Astronomical Society 332, 971.

Braginsky V.B., Kardashev N.S., Polnarev A.G., and Novikov I.D., 1990, Propagation of electromagnetic radiation in a random field of gravitational waves and space radio interferometry. Nuovo Cimento B 105, 1141.

Chandrasekhar S., 1969, Ellipsoidal Figures of Equilibrium. Yale Univ. Press, New Haven

Damour T., Esposito-Farèse G., 1998, Light deflection by gravitational waves from localized sources, Phys. Rev. D 58, 042003.

Doroshkevich A.G., Kotok E.V., Polyudov A.N., Shandarin S.F., Sigov Yu.S., Novikov I.D. 1980, Two-dimensional simulation of the gravitational system dynamics and formation of the large-scale structure of the universe, MNRAS, 192, 321-327

Doroshkevich A.G., Müller V., Retzlaff J., Turchaninov V. 1999, Superlarge-scale structure in N-body simulations, MNRAS 306, 575-591

Faraoni V., 1992, Nonstationary gravitational lenses and the Fermat principle, Astrophys. J. 398, 425-428.

Fridman A.M., Polyachenko V.L., 1985, Physics of Gravitating Systems. Springer Verlag, Berlin

Gorbunov D.S., Rubakov V.A. 2011 Introduction to the Theory of the Early Universe. Cosmological Perturbations and Inflationary Theory. World Scientific Publishing, Singapore

Klypin A. A., Shandarin S. F., 1983, Three-dimensional numerical model of the formation of large-scale structure in the Universe, Monthly Notices of the Royal Astronomical Society, 204, 891-907 
Lai D., Rasio F.A., Shapiro S.L., 1993, Ellipsoidal figures of equilibrium - Compressible models, ApJS, 88, 205

Lai D., Rasio F.A., Shapiro S.L., 1994a, Equilibrium, stability, and orbital evolution of close binary systems ApJ, 423, 344 .

Lai D., Rasio F.A., Shapiro S.L., 1994b, Hydrodynamics of rotating stars and close binary interactions: compressible ellipsoid models, ApJ, 437, 742.

Landau L.D., Lifshitz E.M., 1993, The Classical Theory of Fields. Pergamon, Oxford

Lichtenberg A.J., Lieberman M.A., 1983, Regular and Stochastic motion. Springer-Verlag, New York

Lin C.C., Mestel L., Shu F.H., 1965, The Gravitational Collapse of a Uniform Spheroid, ApJ, $142,1431$.

Lynden-Bell D., 1964, On large-scale instabilities during gravitational collapse and the evolution of shrinking Maclaurin spheroids, ApJ, 139, 1195.

Lynden-Bell D., 1965, On the evolution of frictionless ellipsoids, ApJ, 142, 1648.

Lynden-Bell D., 1967, Statistical mechanics of violent relaxation in stellar system, MNRAS, 136, 101.

Lynden-Bell D., 1996, Consequences of one spring researching with Chandrasekhar Current Science, 70, 789.

Mazets E.P., Golenetskii S.V., Il'inskii V.N., Panov V. N., Aptekar R.L., Gur'yan Y.A., Proskura M.P., Sokolov I.A., Sokolova Z.Ya., Kharitonova T.V., 1981, Catalog of cosmic gamma-ray bursts from the KONUS experiment data, ApESSS, 80, 3, 85, 109

Moiseenko S.G., Bisnovatyi-Kogan G.S., Ardeljan N.V., 2006, A magnetorotational core-collapse model with jets, MNRAS, 370, 501-512

Novikov I.D., 1975, Gravitational radiation from a star that is contracting into a disk. Astron. Zh. 52, 657 ?659 (in Russian); English translation: Novikov, I. D., 1976, Gravitational radiation from a star collapsing into a disk. Sov. Astron. 19, 398?399..

Ostriker J.P., Peebles P.J.E., 1973, A numerical study of the stability of flattened galaxies: or, can cold galaxies survive? ApJ, 186, 467

Polnarev A. G., 1985, Polarization and anisotropy induced in the microwave background by cosmological gravitational waves, Sov. Astron. 29, 607.

Rodrigues H., 2014, On determining the kinetic content of ellipsoidal configurations, MNRAS 440, 1519-1526

Rosensteel G., Tran H.Q., 1991, Hamiltonian dynamics of self-gravitating ellipsoids, ApJ, 366, 30

Seljak U., Zaldarriaga M., 1997, Signature of Gravity Waves in the Polarization of the Microwave Background, Phys. Rev. Lett. 78, 2054.

Shandarin S. F., Zeldovich, Ya. B. 1989 The large-scale structure of the universe: Turbulence, intermittency, structures in a self-gravitating medium, Reviews of Modern Physics, 61, 185-220.

Shapiro S.L., 2004, The secular bar-mode instability in rapidly rotating stars revisited, ApJ, 613, 1213.

Sharif M. and Zaeem Ul Haq Bhatti M., 2013, Stability analysis of restricted non-static axial symmetry, Journal of Cosmology and Astroparticle Physics, Issue 11, article id. 014

Sharif M. and Zaeem Ul Haq Bhatti M., 2014, On the stability of a class of radiating viscous self-gravitating stars with axial symmetry, Astroparticle Physics, 56, 35-41

Sheth R.K., Mo H.J., Tormen G. 2001 Ellipsoidal collapse and an improved model for the number and spatial distribution of dark matter haloes, Monthly Notice of the Royal Astronomical Society, 323, 1.

Thuan T.X., Ostriker J.P. 1974, Gravitational Radiation from Stellar Collapse, ApJ, 191, L105L107.

Vandervoort P.O., 2011, On chaos in the oscillations of galaxies, Mon. Not. R. Astron. Soc. 411, $37-53$

Vandervoort P.O., 2014, On chaos in the pulsations of stars, MNRAS 443, 504-521

Zeldovich Ya. B., 1964, Newtonian and Einsteinian Motion of Homogeneous Matter, Astronomicheskii Zhurnal, 41, 873; English translation: Zeldovich Ya. B., 1965, Newtonian and Einsteinian Motion of Homogeneous Matter, Soviet Astronomy, 8, 700

Zeldovich Ya.B., 1970a, Separation of uniform matter into parts under the action of gravitation, 
Astrofizika, 6, 319-335; English translation: Zeldovich Ya.B., 1970, Fragmentation of a homogeneous medium under the action of gravitation, Astrophysics, 6, 164-174

Zeldovich Ya.B., 1970b, Gravitational instability: An approximate theory for large density perturbations, Astronomy and Astrophysics, 5, 84-89

Zeldovich Ya.B., Novikov I.D., 1967, Relativistic Astrophysics. Nauka, Moscow (in Russian) 